\title{
ON THE STRUCTURE OF ORBIT SPACES OF GENERALIZED MANIFOLDS
}

BY

\author{
GLEN E. BREDON(1)
}

Introduction. In the author's Chapter XV, Fixed point sets and orbits of complementary dimension, of [1], it was shown that if a compact Lie group $G$ acts on a locally separable $n$-dimensional cohomology manifold $M$ over $Z$ $\left(n-\mathrm{cm}_{z}\right)$ of finite covering dimension in such a way that the dimension of the fixed point set $F(G, M)$ is $n-k-1$ near a point $x \in F(G, M)$, where $k$ is the maximal dimension of any orbit, then the orbit space $M / G$ is an $(n-k)$ $\mathrm{cm}_{Z}$ with boundary $F(G, M)$ near $x$ (see Definition 1.3 of $[1$, Chapter XV]). Moreover it was shown that, near $x$, there are exactly two types of orbits, these being the fixed points and the principal orbits. The principal orbits are integral cohomology spheres and there is a cross-section near $x$ for the action of $G$. This theorem (1.4 of [1, Chapter XV]) and its immediate corollary (1.5 of [1, Chapter XV]) will be referred to in the present paper as the CDT (complementary dimension theorem).

The present paper studies the case in which $\operatorname{dim}_{z_{2}} F(G, M)=n-k-2$ near $x$ (see Theorem 1.1 for the precise statement), and, although the results are not nearly as complete as those in the CDT, we obtain that $M / G$ is an $(n-k)-\mathrm{cm}_{Z}$ with boundary near $x$. Using slices we obtain a corollary (1.4) which asserts that in the general case of any action of a compact Lie group $G$ on an $n-\mathrm{cm}_{z} M$, the set $C^{*} \subset M / G$ of points in the orbit space around which $M / G$ is not an $(n-k)-\mathrm{cm}_{z}$ (with or without boundary) is a closed set of dimension at most $(n-k-3)$ (in a certain technical sense). If $k=n-2$, we obtain that $M / G$ is a 2 -manifold with boundary (Corollary 1.5 ), which is the main part of Theorem 11 of [3].

We would like to call attention to Lemmas $2.1,2.2$, and 2.5 as well as Corollary 2.4, which, besides being basic for the present paper, have an independent interest. The technique of the proof of Lemma 5.2 is also of some interest.

As to notation we always denote by $H$ an isotropy subgroup of a point on a principal orbit (that is, $H$ is a minimal isotropy group in the sense that $H$ is conjugate to a subgroup of any other isotropy group). We let $k=\operatorname{dim} G / H$ $=\max \{\operatorname{dim} G(y) \mid y \in M\}$ and let $B=\{y \in M \mid \operatorname{dim} G(y)<k\}$ be the set of points on singular orbits, and $E=\left\{y \in M \mid \operatorname{dim} G(y)=k, G_{y} \times H\right\}$, the set of points on exceptional orbits. For any set $A \subset M$ we denote by $A^{*}$ its image in $M^{*}=M / G$. $T$ will denote a maximal torus of $H$ and $T_{0}$ a maximal torus

Received by the editors April 28, 1960.

( $\left.{ }^{1}\right)$ This research was supported by NSF Grant G-5863. 
of $G$. For a subgroup $K \subset G, K^{0}$ denotes the identity component of $K, N(K)$ denotes the normalizer of $K, Z(K)$ denotes the centralizer of $K$, and $F(K, M)$ $=\{y \mid K(y)=y\}$ denotes the set of fixed points of $K$ on $M$. The natural map $M \rightarrow M / G$ is denoted by $\pi \cdot \operatorname{dim}_{L}(X, x)$ stands for the infimum of $\operatorname{dim}_{L}(U)$ for $U$ ranging over all open neighborhoods of $x$ in $X$, as in [1, Chapter $\mathrm{XV}, \S 1.1$ ].

The definitions of the basic notions that we use will be found in [1]: $\operatorname{dim}_{L} U$ in $\mathrm{I}, 1.2 ; n-\mathrm{cm}_{L}, \mathrm{I}, 3.3$ (also see Chapter XV, 2.2);n-cm $\mathrm{cm}_{L}$ with boundary, $\mathrm{XV}, 1.3 ;=_{L F}, \mathrm{XV}, 2.2$ (which we also use for $Y$ a locally closed subspace of $X$ in general). The cohomology we use is always the Alexander-Spanier cohomology with compact supports.

Familiarity with some of the basic results in [1] (especially Chapters I, IV, V, IX, XIII, and XV) will be assumed. As in the proof of the CDT, we reduce the theorem to the case in which $H$ is finite by studying the action of $N(T) / T$ on $F(T, M)$. Lemma 2.1 then implies that if $H$ is finite then rank $(G) \leqq 2$, and the proof of the theorem is taken up for each of the three cases rank $(G)=0,1$, and 2 in $\S \S 3,4$, and 5 . In the appendix ( $\$ 6)$ we state some theorems that will be used in the proof of our main result. The proofs of these theorems essentially appear elsewhere, but the existing statements of the results are not general enough for our purposes.

1. The main results. The main object of this paper is to prove the following theorem.

TheOREM 1.1. Let $M$ be a locally separable $n-\mathrm{cm}_{z}$ of finite covering dimension. Let $G$ be a compact Lie group acting effectively on $M$, let $H$ be an isotropy group of some point on a principal orbit of $G$, and let $k=\operatorname{dim} G / H$.

If $k>0$ and $\operatorname{dim}_{Z_{2}}(F(G, M), x)=n-k-2$, then $M / G$ is an $(n-k)-c m_{Z}$ with boundary $B^{*} \cup E^{*}$ near $\pi(x)$, and $E^{*}=\varnothing$ near $\pi(x)$ if $G$ is connected.

If $k=0, G \neq G^{+}$(where $G^{+}$denotes the subgroup of $G$ preserving the orienta-

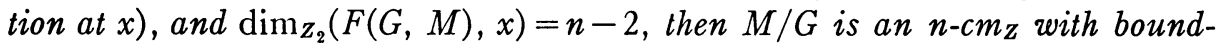
ary $E^{*}$ near $\pi(x)$. Moreover $G$ is dihedral and $G^{+}$is cyclic.

If $k=0, G=G^{+}$, and $\operatorname{dim}_{z_{p}}(F(G, M), x)=n-2$ for all primes $p$ dividing ord $(G)$, then $M / G$ is an $n$-cm near $\pi(x)$. Moreover $G$ is cyclic.

REMARK. In case $\operatorname{rank}(G)>\operatorname{rank}(H)$ one can replace the condition $\operatorname{dim}_{Z_{2}}(F(G, M), x)=n-k-2$ by the weaker $\operatorname{dim}_{Z}(F(G, M), x)=n-k-2$. This will be clear from the proof of 1.1 since the former condition will follow from the latter almost immediately in these cases by using [1, Chapter I, 4.9]. We do not know whether or not the weaker condition suffices in the other cases of 1.1 .

In this section we shall state and prove some corollaries to 1.1 . We will then prove the case $k=0$ of 1.1 and show how this case implies that we may assume that $G$ is connected in the proof of 1.1 .

Analogously with Corollary 1.6 of the CDT, we get the following global result. 
Corollary 1.2. Let $G$ and $M$ be as in 1.1 and also assume $H_{c}^{*}(M, Z)$ $=H_{c}^{*}\left(S^{n}, Z\right)$. If $k>0$, or $k=0$ and $G \neq G^{+}$, and $\operatorname{dim}_{Z_{2}} F(G, M)=n-k-2$, then $M / G$ is a cohomology $(n-k)$-cell with boundary $B^{*} \cup E^{*}$ (see [I, Chapter XV, Corollary 1.6]) and $E^{*}=\varnothing$ if $G$ is connected. If $k=0, G=G^{+}$and $\operatorname{dim}_{z_{p}} F(G, M)$ $=n-2$ for all primes $p$ dividing ord $(G)$, then $M / G$ is a cohomology $n$-sphere, that is, $M / G$ is an $n-c m_{Z}$ and $H_{c}^{*}(M / G, Z)=H_{c}^{*}\left(S^{n}, Z\right)$. In the cases $k=0$, $G=G^{+}$or $G \neq G^{+}, G$ is cyclic or dihedral respectively.

Proof. As in the proof of [1, Chapter XV, Corollary 1.6], we apply 1.1 to the action of $G$ on the cone $c M$ over $M$. The details will be left to the reader.

As in $\left[1\right.$, Chapter IX] let $M_{u, v}=\{y \in M \mid \operatorname{dim} G(y)=u, v=$ number of components of $\left.G_{y}\right\}$.

CoROllary 1.3. With the notation of 1.1 say that $z \in M_{u, v}, H \subset G_{z}$ and $\operatorname{dim}_{L}\left(M_{u, v}^{*}, \pi(z)\right)=n-k-2$, for $L=Z_{2}$ if $u<k$ (resp. for all $L=Z_{p}$ if $u=k$ ), then the hypotheses of 1.1 hold for the action of $G_{z}$ on a slice at $z$, so that $M / G$ is an $(n-k)-c m_{Z}$ with boundary $B^{*} \cup E^{*}$ near $\pi(z)$ if $u<k$ (resp. $M / G$ is an $(n-k)-c m_{Z}$ near $\pi(z)$ with or without boundary if $\left.u=k\right)$.

Proof. This follows exactly as does Corollary 1.5 of [1, Chapter XV].

Corollary 1.4. Let $C^{*}=\left\{z^{*} \in M / G \mid M / G\right.$ is not an $(n-k)-c m_{Z}$ (with or without boundary) near $\left.z^{*}\right\}$. Then $C^{*}$ is a closed set and

(1) $\operatorname{dim}_{Z} C^{*} \leqq n-k-2$,

(2) $\operatorname{dim}_{Z_{2}}\left(C^{*} \cap B^{*}\right) \leqq n-k-3$,

(3) for each $z^{*} \in C^{*} \cap M_{\boldsymbol{k}, 0}^{*}$ there is a prime $p$ (depending on $z$ ) such that $\operatorname{dim}_{z_{p}}\left(M_{k, v}^{*}, z^{*}\right) \leqq n-k-3$.

Proof. $C^{*}$ is clearly closed since its complement is open. (1) follows from Corollary 1.5 of $[1$, Chapter XV] in a manner analogous to the following proof of (2). To prove (2), say that $\operatorname{dim}_{z_{2}}\left(C^{*} \cap B^{*}\right) \geqq n-k-2$ and let $C_{1}^{*}=\left\{z^{*} \in C^{*} \cap B^{*} \mid \operatorname{dim}_{z_{2}}\left(C^{*} \cap B^{*}, z^{*}\right) \geqq n-k-2\right\} . C_{1}^{*}$ is clearly closed and also $\operatorname{dim}_{z_{2}}\left(C_{1}^{*}, z^{*}\right) \geqq n-k-2$ for any $z^{*} \in C_{1}^{*}$. Choose $z^{*} \in C_{1}^{*}$ in such a way that for any $y^{*} \in C_{1}^{*}$ sufficiently close to $z^{*}$ we have $G_{y} \sim G_{z}$. Then if $z^{*} \in M_{u, 0}^{*}$ we see that $C_{1}^{*} \cap M_{u, v}^{*}$ is a neighborhood of $z^{*}$ in $C_{1}^{*}$. Hence $\operatorname{dim}_{z_{2}}\left(M_{u, 0}^{*}, z^{*}\right)$ $\geqq n-k-2$ and it follows from Corollary 1.3 above or from Corollary 1.5 of $\left[1\right.$, Chapter XV] that $M / G$ is an $(n-k)-\mathrm{cm}_{Z}$ (possibly with boundary) near $z^{*}$, contrary to the definition of $C^{*}$. (3) follows immediately from 1.3 or from Corollary 1.5 of $[1$, Chapter XV].

COROLLARY 1.5. If $k=n-2$, then $M / G$ is a 2-manifold with boundary containing $B^{*}$.

Proof. It follows immediately from Corollary 1.4 that $M / G$ is a $2-\mathrm{cm}_{Z}$ with or without boundary. Also it is well-known that a locally separable 
2 -cm is a 2 -manifold. That $B^{*}$ is contained in the boundary follows easily by application of Corollary 1.3 .

This corollary is, of course, just Theorem 11 of [3] for $G$ a Lie group and for $M$ an $n$ - $\mathrm{cm}_{Z}$ instead of a manifold. (The proof in [3] also holds for cms, however.) Similarly the case $k=n-1$ of the CDT would yield immediately a corollary similar to Theorem 10 of [3]. The author intends to give more detailed information about the case $k=n-2$ in a subsequent paper.

We shall now take up the proof of the case $k=0$ of 1.1. We first confine our discussion to the case $G=G^{+}$. In [2, Theorem 7.8], the local groups of $M / G$ are calculated for $G \approx Z_{p}$. It is easy to see from that result that if $G=G^{+} \approx Z_{p}$ and $\operatorname{dim}_{z_{p}}(F(G, M), x)=n-2$, then $M / G$ is an $n-\operatorname{cm}_{z}$ near $\pi(x)$. By an easy induction this follows for all solvable $G=G^{+}$. Thus to show $M / G$ to be an $n-\mathrm{cm}$ it suffices to show that $G$ is solvable. Note that if $K \subset G$ is normal and $K=K^{+}$is solvable then by the diagram ( $Q=$ rationals)

$$
\begin{array}{cc}
H_{c}^{n}(M / K, Q) \stackrel{g K}{\longrightarrow} H_{c}^{n}(M / K, Q) \\
\downarrow \pi^{*} & \downarrow \pi^{*} \\
H_{c}^{n}(M, Q) \stackrel{g}{\longrightarrow} H_{c}^{n}(M, Q)
\end{array}
$$

in which the maps $\pi^{*}$ are isomorphisms by [1, Chapter III, 2.3], we have that $g \in G$ preserves orientation on $M$ if and only if $g K \in G / K$ preserves orientation on $M / K$. Moreover, if $G$ is effective on $M$ then its principal isotropy subgroup is trivial and it follows easily that $G / K$ is effective on $M / K$.

Noting also that if $G=G^{+}$then $G * Z_{p} \oplus Z_{p}$ by dimensional parity and Borel's formula [1, Chapter XIII, 4.3], we see that the class $\delta$ of finite groups $G=G^{+}$which can act effectively on an $n$-cm $M$ with $\operatorname{dim}_{z_{p}}(F(G, M), x)$ $=n-2$ near some $x \in F(G, M)$ and for all primes $p$ dividing ord $(G)$ satisfies the following three statements:

(1) $Z_{p} \oplus Z_{p} \notin S$ for any prime $p$.

(2) If $G \in \mathcal{S}$ and $G^{\prime} \subset G$, then $G^{\prime} \in \mathcal{S}$.

(3) If $G \in \mathcal{S}$ and $K$ is a solvable normal subgroup of $G$, then $G / K \in \mathcal{S}$.

The case $k=0, G=G^{+}$of 1.1 , except for the statement that $G$ is cyclic, now follows from

LEMMA 1.6. Let $\mathrm{S}$ be a class of finite groups satisfying (1), (2) and (3) above. Then every $G \in S$ is solvable and every p-group $G \in S$ is cyclic.

Proof. By $[8$, Chapter $\mathrm{V}, 10]$, it suffices to prove that every p-group $G \in \mathcal{S}$ is cyclic. We use induction on $\operatorname{ord}(G)$. Thus assume all $p$-groups $G^{\prime} \in \mathcal{\delta}$ of order less than $p^{a}$ are cyclic and let $G \in \mathcal{S}$ be a $p$-group of order $p^{a}$. By $[8$, Chapter IV, 9] the center $C$ of $G$ is nontrivial. Also (1) implies that $C$ is cyclic. (3) and the induction hypothesis imply that $G / C$ is cyclic. But if $g$ is a representative of a generator of $G / C$ and $c$ is a generator of $C$, then clearly $G$ is generated by the commuting elements $g$ and $c$. Thus $G$ is abelian and hence $G=C$ is cyclic by (1). 
It remains to show that, in general, $G$ is cyclic. According to Lemma 1.6 and [8, Chapter $\mathrm{V}, 11]$, if $G$ is not cyclic then $G$ has a cyclic normal subgroup $G^{\prime} \approx Z_{a}$ and a subgroup $G^{\prime \prime} \approx Z_{b}$ such that $G^{\prime \prime} \approx G / G^{\prime}$ naturally. Moreover, letting $g$ and $h$ be generators of $G^{\prime}$ and $G^{\prime \prime}$ respectively, $h g h^{-1}=g^{r}$ for some $1<r<a$ and also $((r-1) b, a)=1$ and $r^{b} \equiv 1(a)$. These relations imply that $a$ is odd. Let $p \mid a$ be some odd prime. Then $g^{a / p}$ and $h$ generate a subgroup of $G$ which is abelian if and only if $a$ divides $r(a / p)-(a / p)=(r-1)(a / p)$ and hence if and only if $p \mid(r-1)$ which is impossible, since $(r-1)$ and $a$ are relatively prime. Thus, passing to this subgroup, we may assume that $a=p$ is an odd prime.

A result of R. G. Swan [6, Theorem 3(b)] implies that the p-period of $G$ is $2 t \geqq 4$, where $t$ is the least integer for which $r^{t} \equiv 1(p)$. This means in the present case, since $p \mid \operatorname{ord}(G)$ and $p^{2} \nmid \operatorname{ord}(G)$ and according to [4, Chapter XII, Exercise 11], that

$$
H^{i}(G, Z, p)= \begin{cases}Z_{p}, & \text { if } 2 t \mid i, \\ 0, & \text { otherwise }\end{cases}
$$

where $H^{i}(G, Z, p)$ denotes the $p$-primary component of $H^{i}(G, Z)$. Thus by the universal coefficient theorem

$$
H^{i}\left(G, Z_{p}\right)= \begin{cases}Z_{p}, & \text { if } 2 t \mid i \text { or } 2 t \mid(i+1), \\ 0, & \text { otherwise. }\end{cases}
$$

This could also be seen by direct computation using the methods of $[4$, Chapter XII].

Now let $F=F\left(G^{\prime}, M\right)$, which is an $(n-2)$-cm over $Z_{p}$, and hence coincides with $F(G, M)$ in some open set near $x$. In particular $G$ preserves orientation on $F$. It follows from [1, Chapter IV, 3.6 and Chapter V, 2.1], that if $i>n$ and $V \subset U$ are properly chosen, then in the diagram

$$
\begin{gathered}
H_{c}^{i}\left(V_{G}, Z_{p}\right) \rightarrow H_{c}^{i}\left((V \cap F)_{G}, Z_{p}\right) \\
\downarrow j_{1} \\
H_{c}^{i}\left(U_{G}, Z_{p}\right) \rightarrow H_{c}^{i}\left((U \cap F)_{G}, Z_{p}\right)
\end{gathered}
$$

the horizontal maps are isomorphisms, $\operatorname{Im} j_{1} \approx H^{i-n}\left(G, H_{c}^{n}\left(U, Z_{p}\right)\right)$ $\approx H^{i-n}\left(G, Z_{p}\right)$, and $\operatorname{Im} j_{2} \approx H^{i-(n-2)}\left(G, H_{c}^{n-2}\left(U \cap F, Z_{p}\right)\right) \approx H^{i-n+2}\left(G, Z_{p}\right)$. Thus, for all $j>0, H^{j}\left(G, Z_{p}\right) \approx H^{j+2}\left(G, Z_{p}\right)$, which contradicts our previous result and finishes the proof that $G=G^{+}$is cyclic.

We now take up the case $k=0, G \neq G^{+}$of 1.1 . We first show:

LEMMA 1.7. If $g \in G-G^{+}$, then there is an $i$ such that $\operatorname{dim}_{Z_{2}}\left(F\left(g^{i}, M\right), x\right)$ $=n-1$. Thus $\operatorname{dim}_{L}(F(G, M), x)=n-2$ for any $L$.

Proof. The second statement follows from the first, from the CDT $(k=0)$ (or [7, Lemma 16, Corollary]) which implies that $F\left(g^{i}, M\right)$ is an $(n-1)-\mathrm{cm}$ 
over $Z$ near $x$, and from [1, Chapter $\mathrm{I}, 4.9]$.

To prove the first statement let ord $g=2^{j} a, a$ odd. Then $g^{a}$ also reverses orientation so that we may assume that ord $g=2^{i}$. Let $G^{\prime}$ be the group generated by $g$, ord $G^{\prime}=2^{i}$. By the case $k=0, G=G^{+}$of 1.1 we have that $M / G^{+}$ is an $n-\mathrm{cm}_{z}$ near $x$ and we also have that $g$ must reverse orientation on $M / G^{\prime+}$. Thus since $g^{2} \in G^{\prime+}$ we have that $\operatorname{dim}_{Z_{2}}\left(F\left(g, M / G^{\prime+}\right), x\right)=n-1$. However, if $\operatorname{dim}_{Z_{2}} F\left(g^{i}, M\right)=n-2$ for all $i<2^{j}$ we would have that $G^{\prime}$ acts freely outside $F\left(G^{\prime}, M\right)$ near $x$, since $F(g, M) \subset F\left(g^{i}, M\right)$, both being $(n-2)$-cm's over $Z_{2}$ near $x$, are equal near $x$. It would follow that $G^{\prime} / G^{\prime+}$ acts freely outside $F\left(G^{\prime}, M\right) / G^{\prime+} \approx F\left(G^{\prime}, M\right)$ on $M / G^{\prime+}$ and hence that $F\left(g, M / G^{+}\right) \approx F(g, M)$ is of dimension $n-2$ over $Z_{2}$. This contradiction finishes the proof of the lemma.

By Lemma 1.7 , and the case $k=0, G=G^{+}$of 1.1 , we have that $M / G^{+}$is an $n-\mathrm{cm}_{z}$ near $x$. By the CDT for $k=0$ applied to the action of $G / G^{+}$on $M / G^{+}$we have that $M / G$ is an $n-\mathrm{cm}_{Z}$ with boundary. We must show that the boundary of $M / G$ is $E^{*}$ and that $G$ is dihedral. There is an invariant neighborhood $U$ of a point $y \in F(G, M)$ arbitrarily near $x$, such that $F(g, U)$ $=F(G, U)=F$ for all $e \neq g \in G^{+}$. We may assume that $U$ is so small that if $z \in U, G_{z} \neq(e), G$, then $G_{z} \approx Z_{2}$ and $F\left(G_{z}, U\right)$ is an $(n-1)$-cmz. It is also possible to assume that if $C^{*}$ is the boundary of $U / G$, then $F^{*} \subset C^{*}$ is connected, $C^{*}-F^{*}$ has exactly two components (since $F$ is an $(n-2)-\mathrm{cm}_{z_{p}}$ for any $p \mid$ ord $\left.G^{+}\right) C_{0}^{*}$ and $C_{1}^{*}$, and $U^{*}-C^{*}$ is connected.

It follows from Theorem 6.5 that $U^{*}$ may be taken to be so small that $\pi^{-1}\left(U^{*}-C^{*}\right)$ consists of ord $(G)$ components each mapping homeomorphically onto $U^{*}-C^{*}$ via $\pi$. Let $W$ be one of these components.

Note that since $F$ is an $(n-2)-\mathrm{cm}_{z_{p}}$ for any $p$ ord $G^{+}$we obtain from the cohomology sequence of $C^{*} \bmod F^{*}$ that $H_{c}^{n-2}\left(C_{i}^{*}, Z_{p}\right)={ }_{L F} 0$ for $i=0,1$ and any $p \mid$ ord $G^{+}$. Also, if $z \in \pi^{-1}\left(C_{i}^{*}\right)$, then $G^{+}(z)=G^{+} G_{z}(z)=G(z)$ so that

$$
\frac{\pi^{-1}\left(C_{i}^{*}\right)}{G^{+}} \approx C_{i}^{*} \text {. }
$$

Thus, again by Theorem 6.5 , we obtain that $\pi^{-1}\left(C_{i}^{*}\right)$ consists of ord $\left(G^{+}\right)$ $=(1 / 2) \operatorname{ord}(G)$ components. Let $C_{i}$ be a component of $\pi^{-1}\left(C_{i}^{*}\right)$ such that $C_{i} \cap \bar{W} \neq \varnothing$. We see easily, using the connectivity of $C_{i}$, that $C_{i} \subset \bar{W}$. It follows that $\bar{W}=W \cup C_{0} \cup C_{1} \cup F$. Note that $G_{z}$ is constant on $C_{0}$ and on $C_{1}$ and hence if $z \in C_{0}$ then $C_{0} \subset F\left(G_{z}, U\right)$.

We can construct in $\bar{W}$ an arc $\alpha:[0,1] \rightarrow \bar{W}$ such that $\alpha(a) \in W$ for $a \in(0,1), z_{0}=\alpha(0) \in C_{0}$ and $z_{1}=\alpha(1) \in C_{1}$. Define $g_{1}$ to be the nontrivial element of $G_{z_{1}}$ and let $g_{2}$ be the nontrivial element of $G_{g_{1}\left(z_{0}\right)}$. In general define $g_{2 i}$ to be the nontrivial element of $G_{g_{2 i-1} g_{2 i-z}} \cdots g_{8} g_{1}\left(z_{0}\right)$ and $g_{2 i+1}$ to be the nontrivial element of $G_{g_{2 i} \theta_{i-2} \cdots g_{4 g_{2}\left(z_{1}\right)} \text {. }}$

We see easily that $U_{j}\left\{g_{j} g_{j-1} \cdots g_{2} g_{1}(\bar{W}-F)\right\}$ is connected and is open and closed in $U-F$. Thus it is equal to $U-F$ and this implies that 
$\bigcup_{j}\left\{g_{j} g_{j-1} \cdots g_{2} g_{1}\right\}=G$. Now $\bigcup_{j}\left\{\left(g_{j} g_{j-1} \cdots g_{2} g_{1}\right) \alpha([0,1])\right\}$ must be a circle (since it is a compact connected one-manifold) and hence $\gamma=G(\alpha([0,1]))$ is a circle upon which $G$ operates effectively (since the isotropy group is trivial on $\alpha(0,1))$. It follows that $G$ is dihedral.

Now let $V$ be an arbitrary invariant neighborhood of $x$ such that the complement $X$ in $V / G$ of the boundary (as an $n$-cm with boundary) of $V / G$ is connected and assume that $V$ is so small that for any sufficiently small neighborhood $V^{\prime} \subset V$ of $x$ and any element $g \in G$ with $\operatorname{dim} F(g, V)=n-1$, then there are exactly two components of $V-F(g, V)$ which touch $V^{\prime}$ (and these are permuted by $g$ ).

In some such $V^{\prime}$ we can find a $U$ as above and $\gamma \subset U$. Say that $a, b \in \gamma$ are in different components of $\gamma-F(g, \gamma)$. Then clearly $a=g\left(b^{\prime}\right)$ for some $b^{\prime}$ in the same component as $b$. By the selection of $V$ and $V^{\prime}, a$ and $b^{\prime}$ (hence $a$ and $b)$ are in different components of $V-F(g, V)$. Thus we see that $\pi^{-1}(X)$ has at least ord $(G)$ components, since $\gamma \cap \pi^{-1}(X)$ has this many components and since any two points in different components of $\gamma-\pi^{-1}(X)$ are actually in different components of $\gamma-F(g, \gamma)$ for some $g \in G$.

However, the number of components of $\pi^{-1}(X)$ is at most the number of elements of $G(z)$ for any $z$ for which $\pi(z) \in X$. Thus, for any such $z, G(z)$ has as many points as $G$, that is, $G(z)$ is principal. This concludes the proof of the case $k=0, G \neq G^{+}$of 1.1 .

The case $k=0$ enables us to reduce the proof of Theorem 1.1 to the connected case as follows. Say that $(G, M)$ is a transformation group satisfying the hypotheses of 1.1. Then $\left(G^{0}, M\right)$ also satisfies these hypotheses (or the hypotheses of the CDT) so that $M / G^{0}$ is an $(n-k)-\mathrm{cm}_{z}$ with boundary $B / G^{0}$. We now apply the case $k=0, G \neq G^{+}$to the action of $G / G^{0} \oplus K$ on $\left(M / G^{0}\right)^{d\left(B / G^{0}\right)}$ (see [1, Chapter XV, Definition 1.2]), where $K$ is induced by the doubling operation. We obtain that

$$
M / G \approx \frac{M / G^{0}}{G / G^{0}} \approx \frac{\left(M / G^{0}\right)^{d\left(B / G^{0}\right)}}{\left(G / G^{0}\right) \oplus K}
$$

is an $(n-k)-\mathrm{cm}_{Z}$ with boundary. The boundary is clearly $B^{*} \cup E^{*}$ by the results of the case $k=0, G \neq G^{+}$of 1.1 . Thus we may restrict our attention to the case in which $G$ is connected in the proof of Theorem 1.1.

REMARK. A closer examination of the action of $G / G^{0}$ on $M / G^{0}$ yields that (when $k>0$ ) effectively $G / G^{0}$ is either trivial or $Z_{2}$. Also the former case implies that the orbits of $G$ are connected (and, in particular, $E=\varnothing$ near $x$ since this is true for $G^{0}$ ), and the latter case implies that the boundary of $M / G$ is $B^{*} \cup E^{*}$ with $B^{*} \cap \overline{E^{*}}$ an $(n-k-2)-\mathrm{cm}_{z}$. To see this, assume $G / G^{0}$ does not act trivially on $M / G^{0}$, let $G^{\prime}$ be the effective (factor) group, and let $B^{\prime}=B / G^{0}$ be the boundary of $M^{\prime}=M / G^{0}$. Then $G^{\prime}$ is effectively of order two on $B^{\prime}$ since its fixed point set is of dimension $=n-k-2=\operatorname{dim} B^{\prime}-1$. Since any group leaving $B^{\prime}$ stationary must also leave $M^{\prime}$ stationary (see $[1$, Chapter $\mathrm{XV}$, 
remark below 2.1]) we see that $G^{\prime} \approx Z_{2}$. The rest follows from the fact that $\overline{E^{*}} \approx F\left(G^{\prime}, M^{\prime}\right)=F\left(G / G^{0}, M / G^{0}\right)$ and $\overline{E^{*}} \cap B^{*} \approx F\left(G / G^{0}, B / G^{0}\right)$. (Also compare Lemma 2.5.)

2. Preliminary results. Following the method of $[1$, Chapter XV], we shall reduce the proof of Theorem 1.1 to the case in which $H$ is finite by studying the action of $N(T) / T$ on $F(T, M)$, where $T$ is a maximal torus of $H$. Our first lemma gives a bound for $\operatorname{rank}(N(T) / T)=\operatorname{rank}(G)-\operatorname{rank}(H)$ and is an improvement of the technique used in [1, Chapter XV]. The lemma is also an improvement of the known inequalities $\operatorname{dim}_{z} F(G, M) \leqq n-k-1$ and $\operatorname{dim}_{Z} F\left(T_{0}, M\right) \leqq n-2\left[\operatorname{rank}\left(T_{0}\right)\right]$ where $T_{0}$ is the maximal torus of $G$.

LeMma 2.1. Let $G$ act effectively on a locally separable $n$-cm $M$ with principal isotropy group $H$ and $k=\operatorname{dim} G / H$. Then near a point $x \in F(G, M)$ we have $\operatorname{dim}_{Z} F(G, M) \leqq \operatorname{dim}_{Z} F\left(T_{0}, M\right) \leqq n-k-(\operatorname{rank}(G)-\operatorname{rank}(H)) \leqq n-2$ $\operatorname{rank}(G)$.

Proof. Let $r=\operatorname{rank}(G)-\operatorname{rank}(H)$. The first inequality is clear. We now prove the second inequality. It suffices to consider the case in which $H$ is finite, since if $T$ is a maximal torus of $H$ we have that near $x$ (see the proof of Lemma 2.7 of $[1$, Chapter XV])

$$
\operatorname{dim}_{Z} F(T, M)=n-k+\operatorname{dim} \frac{N(T)}{N(T) \cap H}
$$

and the lemma for $H$ finite says that near $x$

$$
\begin{aligned}
\operatorname{dim}_{Z} F\left(T_{0}, M\right) & \leqq\left(n-k+\operatorname{dim} \frac{N(T)}{N(T) \cap H}\right)-\operatorname{dim} \frac{N(T)}{N(T) \cap H}-r \\
& =n-k-r .
\end{aligned}
$$

Note also that it suffices to prove the inequality for $G$ connected.

We shall assume from now on that $H$ is finite and hence that $r=\operatorname{rank}(G)$. If $r=0$ the inequality is clear. If $r=1$ then the natural map

$$
\frac{F\left(T_{0}, M\right)}{N\left(T_{0}\right)} \rightarrow M / G
$$

is a homeomorphism into $B^{*}$. Thus by [1, Chapter IX, 2.2 Corollary],

$$
\operatorname{dim} F\left(T_{0}, M\right)=\operatorname{dim} \frac{F\left(T_{0}, M\right)}{N\left(T_{0}\right)} \leqq \operatorname{dim} B^{*} \leqq n-k-1
$$

as was to be shown. Thus assume $r>1$.

We shall prove the inequality by induction on $n=\operatorname{dim} M$. If $n=0$ the inequality is trivial. Say that the inequality always holds for $n<n_{0}$ and let $\operatorname{dim} M=n_{0}$. First suppose that $F\left(T_{0}, M\right)$ does not coincide with $F(G, M)$ in any open set near $x$. Then let $y$ be a point of the component of $F\left(T_{0}, M\right)$ 
containing $x$, such that $y \notin F(G, M)$ and such that for $y^{\prime} \in F\left(T_{0}, M\right)$ near $y, G_{y} \sim G_{y^{\prime}}$. Let $S$ be a slice at $y$. Then by the inductive hypothesis $\operatorname{dim} F\left(T_{0}, S\right)$ $\leqq\left(n_{0}-\operatorname{dim} G / G_{y}\right)-\operatorname{dim} G_{y}-r=n_{0}-k-r$. If $z \in F\left(T_{0}, G(S)\right)$ and $z^{\prime} \in S \cap G(z)$ then $G_{z^{\prime}} \subset G_{y}$ is of maximal rank so that there is an element $g \in G_{y}$ such that $G_{g\left(z^{\prime}\right)}=g G_{z^{\prime}} g^{-1}$ contains $T_{0}$. Hence $g\left(z^{\prime}\right) \in F\left(T_{0}, S\right) \cap G(z)$ and it follows that $F\left(T_{0}, S\right)^{*}=F\left(T_{0}, G(S)\right)^{*}$. Thus, since $y$ is near $x$, we have $\operatorname{dim} F\left(T_{0}, S\right)$ $=\operatorname{dim} F\left(T_{0}, S\right)^{*}=\operatorname{dim} F\left(T_{0}, G(S)\right)^{*}=\operatorname{dim} F\left(T_{0}, G(S)\right)=F\left(T_{0}, M\right)$ and hence

$$
\operatorname{dim} F\left(T_{0}, M\right) \leqq n_{0}-k-r
$$

as was to be shown.

Now say that $F\left(T_{0}, M\right)$ coincides with $F(G, M)$ in some open set near $x$. Then in order to compute $\operatorname{dim} F\left(T_{0}, M\right)$ we may assume that $F\left(T_{0}, M\right)$ $=F(G, M)$ near $x$. By Borel's formula [1, Chapter XIII, 4.3] there is a $T_{1} \subset T_{0}$, rank $T_{1}=r-1$, such that near $x, \operatorname{dim} F\left(T_{1}, M\right)>\operatorname{dim} F\left(T_{0}, M\right)$. Let $y \in F\left(T_{1}, M\right)-F\left(T_{0}, M\right)$ be in the component of $F\left(T_{1}, M\right)$ containing $x$ and assume that for $y^{\prime} \in F\left(T_{1}, M\right)$ close to $y ; G_{y^{\prime}} \sim G_{y}$. Since $F\left(T_{0}, M\right)=F(G, M)$ we may assume that rank $G_{y}=r-1$, that is, that $T_{1}$ is a maximal torus of $G_{y}$. Let $S$ be a slice at $y$. Then by the inductive assumption

$$
\begin{aligned}
\operatorname{dim} F\left(T_{1}, S\right) & \leqq\left(n_{0}-\operatorname{dim} G / G_{y}\right)-\operatorname{dim} G_{y}-r+1 \\
& =n_{0}-k-r+1 .
\end{aligned}
$$

Since rank $G_{y}=r-1$ we have that $F\left(T_{1}, S\right) /\left(N\left(T_{1}\right) \cap G_{y}\right) \approx F\left(T_{1}, S\right)$ * so that $\operatorname{dim} F\left(T_{1}, S\right)=\operatorname{dim} F\left(T_{1}, S\right)^{*}$. Just as above we see easily that

$$
F\left(T_{1}, S\right)^{*}=F\left(T_{1}, G(S)\right)^{*} \approx \frac{F\left(T_{1}, G(S)\right)}{N\left(T_{1}\right)} .
$$

But $N\left(T_{1}\right)$ acting on $F\left(T_{1}, M\right)$ or $F\left(T_{1}, G(S)\right)$ is effectively of dimension equal to $\operatorname{dim} N\left(T_{1}\right) / T_{1}$ so that

$$
\begin{aligned}
\operatorname{dim} F\left(T_{1}, M\right) & =\operatorname{dim} F\left(T_{1}, G(S)\right)=\operatorname{dim} \frac{F\left(T_{1}, G(S)\right)}{N\left(T_{1}\right)}+\operatorname{dim} \frac{N\left(T_{1}\right)}{T_{1}} \\
& \leqq n_{0}-k-r+1+\operatorname{dim} \frac{N\left(T_{1}\right)}{T_{1}} .
\end{aligned}
$$

Also $N\left(T_{1}\right)$ is effectively of rank one on $F\left(T_{1}, M\right)$ and hence by the inequality for $r=1$ which has already been proved we have

$$
\begin{aligned}
\operatorname{dim} F\left(T_{0}, M\right)= & \operatorname{dim} F\left(T_{0}, F\left(T_{1}, M\right)\right) \leqq\left(n_{0}-k-r+1+\operatorname{dim} \frac{N\left(T_{1}\right)}{T_{1}}\right) \\
& -\operatorname{dim} \frac{N\left(T_{1}\right)}{T_{1}}-1=n_{0}-k-r
\end{aligned}
$$

as was to be shown. 
The last inequality can be derived from the middle one as follows. Note that it is equivalent to $k \geqq \operatorname{rank}(G)+\operatorname{rank}(H)$. We consider the action of $T$ on $G / H$. This action is effective since $G$ is effective on $G / H$ (for otherwise $H$ would contain a subgroup $K$ normal in $G$ and we would have that $K$ acts trivially on $M)$. Thus $\operatorname{rank}(G)-\operatorname{rank}(H) \leqq \operatorname{dim} N(T) /(N(T) \cap H)$ $=\operatorname{dim} N(T) H / H=\operatorname{dim} F(T, G / H) \leqq k-\operatorname{dim}(T)-\operatorname{rank}(T)=k-2 \operatorname{rank}(H)$, by the middle inequality applied to the action of $T$ on $G / H$. Thus $k \geqq \operatorname{rank}(G)$ + rank $(H)$ as was to be shown.

REMARK. Notice that the proof of the middle inequality implies that if we have equality for the first two inequalities and $H$ is finite, then, since $F\left(T_{0}, M\right)=F(G, M)$ in some open set near $x$, we would have that for any $T_{1} \subset T_{0}$ of codimension one, either $\operatorname{dim}\left(F\left(T_{1}, M\right), x\right)=\operatorname{dim}\left(F\left(T_{0}, M\right), x\right)$ $=n-k-r$ or $\operatorname{dim}\left(F\left(T_{1}, M\right), x\right)=n-k-r+1+\operatorname{dim} N\left(T_{1}\right) / T_{1}$.

REMARK. In the situation of the main theorem (1.1), Lemma 2.1 allows us to obtain the bound $(\operatorname{rank}(G)-\operatorname{rank}(H)) \leqq n-k-\operatorname{dim} F(G, M)=(n-k)$ $-(n-k-2)=2$, and hence $\operatorname{rank}(G) \leqq 2$ when $H$ is finite.

The next lemma is an improvement of Lemma 2.5 of [1, Chapter XV]. It was implicit in some of the proofs there, but was not explicitly stated.

Leмma 2.2. Let $G$ act on the $n-\mathrm{cm}_{z} M$ and assume that $M$ is such that the map $F(T, M) / N(T) \rightarrow M / G$ is a homeomorphism (see [1, Chapter XV, Lemma 2.7]). Let $z \in F(T, M)$. If $N(T)(z)$ is principal for $N(T)$ on $F(T, M)$ then $G(z)$ is principal for $G$ on $M$.

Proof. There is an element $g \in G$ such that $g^{-1} H g \subset G_{z}$. Thus $H \subset g G_{z} g^{-1}$ $=G_{g(z)}$ so that $g(z) \in F(H, M)$ and $g(z)$ and $z$ are on the same orbit of $N(T)$ on $F(T, M)$ since they are on the same orbit of $G$ and are contained in $F(T, M)$. Thus we may assume that $H \subset G_{z}$ so that $z \in F(H, M)$.

The hypothesis implies that $N(T) \cap G_{z}=N(T) \cap H$ since the latter group is a principal isotropy group for $N(T)$ on $F(T, M)$. Let $S$ be a slice at $z$ for $G$ on $M$ so that by Lemma 2.7 of $[1$, Chapter XV] any sufficiently small neighborhood $S^{\prime}$ of $z$ in $F(T, S)$ is a slice for the action of $N(T)$ on $F(T, M)$. Let $z^{\prime} \in S^{\prime}$. Then since $z$ is on a principal orbit of $N(T)$ we must have

$$
N(T) \cap G_{z^{\prime}}=N(T) \cap G_{z}=N(T) \cap H .
$$

Hence

$$
\varnothing \neq F\left(H, G_{z} / G_{z^{\prime}}\right) \subset F\left(T, G_{z} / G_{z^{\prime}}\right) \approx \frac{N(T) \cap G_{z}}{N(T) \cap G_{z^{\prime}}}=\frac{N(T) \cap H}{N(T) \cap H}=\text { a point. }
$$

Thus $F\left(H, G_{z}\left(z^{\prime}\right)\right)=F\left(T, G_{z}\left(z^{\prime}\right)\right)=z^{\prime}$, since $T \subset G_{z^{\prime}}$, for any $z^{\prime} \in S^{\prime}$. This implies that $F\left(H, S^{\prime}\right)=F\left(T, S^{\prime}\right)=S^{\prime}$ is a cross-section near $z$ for the orbit map $F(T, M) \rightarrow F(T, M) / N(T)$ and hence also for the orbit map $M \rightarrow M / G$. The lemma now follows from [1, Chapter $\mathrm{XV}, 2.6]$. 
REMARK. Note that by Lemma 2.7 of [1, Chapter XV] we may assume, in the proof of Theorem 1.1, that the natural map $F(T, M) / N(T) \rightarrow M / G$ is a homeomorphism. Moreover, since the finite group $(N(T) \cap H) / T$ is a principal isotropy group for the action of $N(T) / T$ on $F(T, M)$, it follows from Lemma 2.2 that if $\operatorname{rank}(G)>\operatorname{rank}(H)$ (that is, if $\operatorname{dim}(N(T) / T)>0$ ), then it suffices to prove Theorem 1.1 for the action of $N(T) / T$ on $F(T, M)$ (made effective) and hence for the case in which $H$ is finite. (The fact that $E^{*}=\varnothing$ near $\pi(x)$ when $G$ is connected does not follow directly, but is easily obtained by the method used in the last paragraph of $\S 3$.) In case $\operatorname{rank}(G)$ $=\operatorname{rank}(H)$ there are some minor complications due to the essential difference between the cases $k>0$ and $k=0$ of Theorem 1.1. These complications are dealt with in $\$ 3$.

LEMmA 2.3. If $G$ acts on a locally separable $n-c m_{Z} M$ of finite covering dimension in such a way that there are no exceptional orbits and such that $\operatorname{dim}_{Z}\left(F\left(G^{0}, M\right), x\right)=n-k-1, x \in F(G, M)$, then the orbits of $G$ on $M$ are connected. In particular $F(G, M)=F\left(G^{0}, M\right)$.

Proof. By hypothesis the transformation group $\left(G^{0}, M\right)$ satisfies the hypotheses of the CDT so that $M / G^{0}$ is an $(n-k)-\mathrm{cm}$ with boundary $F\left(G^{0}, M\right)$. Also the points of $F\left(G^{0}, M\right)$ are the only singular orbits of $G^{0}$ and hence of $G$. Thus $F\left(G^{0}, M\right)=B$. Let $G / K, G^{0} \subset K \subset G$, be the effective group acting on $M / G^{0}$. Since there are no exceptional orbits of $G$ on $M$, the set $M-B$ of principal orbits is connected. Since the isotropy groups of two nearby points of $M-B$ are conjugate by an element of $G^{0}$ we see that this is true for any two points of $M-B$. If $G_{y}=H$ it is easy to see that the isotropy subgroup of $G$ of the point $G^{0}(y) \in M / G^{0}$ is $G_{0} H$. It follows that $G_{0} H$ leaves pointwise fixed the dense set $(M-B) / G^{0} \subset M / G^{0}$ and hence, in fact, $K=G_{0} H$. Since we also have that if $G_{y^{\prime}} \sim H$ (by an element of $G^{0}$ ) then $G^{0} G_{y^{\prime}}=G^{0} H$ we see that the points of $(M-B) / G^{0}$ are all on principal orbits of $G / K$ on $M / G^{0}$.

Let $g \in G$ be such that ord $(g K)=p$ in $G / K$, p prime. Since the points of $\left(M-F\left(G^{0}, M\right)\right) / G^{0}=(M-B) / G^{0}$ are principal for $G / K$ (and hence have trivial isotropy groups) we see that $F\left(g, M / G^{0}\right) \subset F\left(G^{0}, M\right)=$ Boundary of $M / G^{0}$. By the cohomology diagram

$$
\begin{aligned}
& H_{c}^{n-k-1}\left(M / G^{0}\right) \rightarrow H_{c}^{n-k-1}\left(B / G^{0}\right) \rightarrow H_{c}^{n-k}\left(\frac{M-B}{G^{0}}\right) \rightarrow H_{c}^{n-k}\left(M / G^{0}\right) \\
& \begin{array}{cccc}
\downarrow g^{*} & \downarrow g^{*} & \downarrow g^{*} & \downarrow g^{*} \\
H_{c}^{n-k-1}\left(M / G^{0}\right) & \rightarrow H_{c}^{n-k-1}\left(B / G^{0}\right) & \rightarrow H_{c}^{n-k}\left(\frac{M-B}{G^{0}}\right) \rightarrow H_{c}^{n-k}\left(M / G^{0}\right)
\end{array}
\end{aligned}
$$

in which the groups in the first and last columns are $={ }_{L x} 0$ (see $[1$, Chapter $\mathrm{XV}$, Lemma 2.3]) we see easily that $g$ preserves orientation on $B / G^{0}$ $=F\left(G^{0}, M\right)$ if and only if it does so on $(M-B) / G^{0}$ (and hence on $\left.\left(M / G^{0}\right)^{d\left(B / G^{0}\right)}\right)$. 
Thus by dimensional parity we see that if $r=\operatorname{dim}_{Z_{p}}\left(F\left(g, M / G^{0}\right)\right)$, then $(n-k-1)-r$ is even if and only if $(n-k)-r$ is even.

This contradiction shows that $G / G^{0}$ must act trivially on $M / G^{0}$ and hence that the orbits of $G$ are connected as claimed.

Corollary 2.4. Say that $G$ acts on a locally separable $n-c m_{Z} M$ of finite covering dimension. Assume that $x \in B$ and that all orbits of points in $B$ sufficiently close to $x$ are of the same dimension and that $\operatorname{dim}_{Z}\left(B^{*}, \pi(x)\right)=n-k-1$. Then if there are no exceptional orbits near $x$, the transformation group $(G, M)$ satisfies the hypotheses of the CDT [1, Chapter XV, Corollary 1.5] near $x$ so that all the orbits in $B$ near $x$ are of the same type.

Proof. This follows immediately by application of Lemma 2.3 to the action of $G_{x}$ on a slice at $x$. (See, for example, the proof of [1, Chapter XV, 1.5].)

Lemma 2.5. Let $X$ be an $m$-cm over $Z_{2}$ with boundary $B$. Say that $G \approx Z_{2}$ acts on $X$ with $x \in B \cap F(G, X)$. Suppose $\operatorname{dim}_{z_{2}}(F(G, B), x)=r$. Then locally at $x, F(G, X)$ is an $(r+1)-c m_{z_{2}}$ with boundary $F(G, B)$. If $r=m-2$ then locally at $x, X$ is the union of two subsets $X_{1}$ and $X_{2}$ with $X_{1} \cap X_{2}=F(G, X)$, each of which is a cross-section for the action of $G$ and for which $X_{i}$ is an m-cm $Z_{2}$ with boundary $\left(X_{i} \cap B\right) \cup F(G, X)$.

Proof. Let $M=X^{d B}$ and let $h$ be the doubling operation. Let $g$ be the nontrivial element of $G$ and let it act on $M$ in the natural manner. The transformations $e, g, h, g h$ form a group $K$ isomorphic to $Z_{2} \oplus Z_{2}$ acting on $M$. Note that $g h$ interchanges the components of $M-B$ so that $F(g h, M) \subset B=F(h, M)$ and it follows that $F(g h, M)=F(K, M)=F(g, B)$. By Borel's formula [1, Chapter XIII, 4.3]

$$
m-r=((m-1)-r)+(r-r)+\left(\operatorname{dim}_{z_{2}} F(g, M)-r\right)
$$

so that $F(g, M)$ is an $(r+1)-\operatorname{cm}_{z_{2}}$ near $x$. Clearly $h$ acts on $F(g, M)$ with $F(h, F(g, M))=F(g, B)$ and $F(g, X) \approx F(g, M) /(e, h)$. From known facts it follows that $F(g, X)$ is an $(r+1)-\mathrm{cm}_{z_{2}}$ with boundary $F(g, B)=F(h, F(g, M))$.

If $r=m-2$, then from known facts $M$ is the union, near $x$, of subsets $M_{1}, M_{2}$ with $g$ interchanging $M_{1}$ and $M_{2}$, and with $M_{1} \cap M_{2}=F(g, M)$. It follows that $X=M / h$ is the union $X_{1} \cup X_{2}, X_{i}=M_{i} / h, X_{1} \cap X_{2}=F(g, X)=F$. Let $B_{i}=X_{i} \cap B$ which are $(m-1)$-cm's with boundary $F(g, B)=F \cap B$. The Mayer-Vietoris sequence with coefficients in $Z_{2}$,

$$
\rightarrow H_{c}^{j}(X) \rightarrow\left(H_{c}^{j}\left(X_{1}\right) \oplus H_{c}^{j}\left(X_{2}\right)\right) \rightarrow H_{c}^{j}\left(X_{1} \cap X_{2}\right) \rightarrow
$$

implies that $H_{c}^{\prime}\left(X_{i}\right)={ }_{L \nu} 0$ for $y \in F(g, B)$ and this is also true for $y \in\left(B_{i} \cup F\right)$ - $\left(F \cap B_{i}\right)$ since $X_{i}$ is an $m-\mathrm{cm}_{z_{2}}$ with boundary near such points. Thus $H_{c}^{j}\left(X_{i}\right)_{-\left(L B_{i} \cup F\right)} 0$ for all $j$. Moreover $B_{i} \cup F$ is the union of the two $(m-1)$ -cm's $B_{i}$ and $F$ with intersection $B_{i} \cap F=F(g, B)$ the boundary of each. Thus $B_{i} \cup F$ is an $(m-1)-\mathrm{cm}_{Z_{2}}$ and the conclusion of the lemma follows from [1, 
Chapter XV, 2.3] (which is also valid for coefficients in a field).

We will need the following lemma on cm's with boundary. We understand that F. Raymond has been able to prove a more general result in this direction.

Leмма 2.6. Let $X$ be a locally compact space and $F \subset B \subset X$, closed subspaces. Suppose that the following conditions hold:

(1) $X-F$ is an $m-c m_{Z}$ with boundary $B-F$.

(2) $B$ is an $(m-1)-c m_{Z}$.

(3) $\operatorname{dim}_{z} F \leqq m-2$.

(4) For all $x \in F, H_{c}^{i}(X-B, Z)={ }_{L x} \begin{cases}Z, & i=m, \\ 0, & i \neq m .\end{cases}$

Then $X$ is an $m-c m_{Z}$ with boundary $B$.

Proof. According to Lemma 2.3 of [1, Chapter XV] we must show that $H_{c}^{i}(X, Z)=_{L B} 0$ for all $i$ and by (1) this reduces to showing that $H_{c}^{i}(X, Z)$ $={ }_{L x} 0$ for any $x \in F$ and all $i$. By the cohomology sequences of $X \bmod B$ (locally about $x$ ) it clearly suffices to show that for a fundamental system of neighborhoods $U$ of $x$, the connecting homomorphism $H_{c}^{m-1}(U \cap B, Z)$ $\rightarrow H_{c}^{m}(U-B, Z)$ is an isomorphism onto.

By (2) and (4), since $X-B$ is an $m-\mathrm{cm}$, we see that it can be assumed that $U \cap B$ and $U-B$ are connected and orientable. Let $y \in U \cap B-F$ (which exists by (3)) and let $V \subset U$ be a neighborhood of $y$ such that $V \cap B \subset B-F$ is connected and $V-B C U-B$ is connected. Then, since $H_{c}^{m}(V)=0$ by (1) ( $V$ is a proper closed subset of the $\left.m-\mathrm{cm} V^{d(B \cap V)}\right)$, we have that $Z \approx H_{c}^{m-1}(V \cap B) \rightarrow H_{c}^{m}(V-B) \approx Z$ is onto and hence is an isomorphism. But $H_{c}^{m-1}(V \cap B) \rightarrow H_{c}^{m-1}(U \cap B)$ and $H_{c}^{m}(V-B) \rightarrow H_{c}^{m}(U-B)$ are also isomorphisms and it follows that $H_{c}^{m-1}(U \cap B) \rightarrow H_{c}^{m}(U-B)$ is an isomorphism as was to be shown.

3. Case I. rank $(G)=\operatorname{rank}(H)$. In this case $N(T)$ acts on $F(T, M)$ effectively as a finite group and $\operatorname{dim} F(T, M)=n-k$.

We will first show that there is an element $g \in N(T)$ which reverses the orientation of $F(T, M)$ (near $x)$. If not, then we see that $\operatorname{dim} F(g, F(T, M)$ ) $\leqq n-k-2$ for all $g \in N(T)$ (by dimensional parity). It follows that, near $x$, the set $U$ of points on principal orbits of $N(T)$ on $F(T, M)$ forms a dense connected set in $F(T, M)$. At least one of these points has $H$ as an isotropy group (for $G$ on $M$ ) and since $F(H, U) \subset F(T, U)$ must touch all the nearby orbits it follows from the connectivity of $U$ that $F(H, U)=F(T, U)$ (and also that $F(H, M)=F(T, M))$ near $x$.

We now have that $N(H) / H=F(H, G / H)=F(T, G / H)=N(T) H / H$ so that $N(H)=N(T) H$ and hence $N(H) \supset N(T)$. Thus $G / N(H)$ is acyclic over the rationals and is, in particular, nonorientable. Considering the fibering $U \rightarrow^{F(H, U)} G / N(H)$ (see $[1$, Chapter XII, 1.3(2)]) we see that there must be 
an element of the structural group which reverses the orientation of $F(H, U)$ $=F(T, U)$. But this structural group is just $N(H) / H=N(T) H / H$. This implies, of course, that there is an element $g \in N(T)$ reversing the orientation of $F(T, U)$ and hence of $F(T, M)$ as was to be shown.

By the case $k=0, G \neq G^{+}$of Theorem 1.1, and by Lemma 2.2, we now have that $M / G$ is an $(n-k)-\mathrm{cm}_{z}$ with boundary $B^{*} \cup E^{*}$. It remains to show that if $G$ is connected, then $E^{*}=\varnothing$ near $x^{*}$. But if $z^{*} \in E^{*}$, then, since $E^{*}$ is open in $B^{*} \cup E^{*}$, we have $\operatorname{dim}_{z} E^{*}=n-k-1$ and hence we would have $\operatorname{dim}_{Z} E=n-1$ contrary to Theorem 4 of [5]. This completes the proof of Case I.

4. Case II. $\operatorname{rank}(G)=\operatorname{rank}(H)+1$. It will suffice to consider the case in which $H$ is finite and $G$ is connected, and hence for which $\operatorname{rank}(G)=1$. If $G=\mathrm{SO}(2)$ then $\operatorname{dim} F(G, M)$ could not be $n-k-2=n-3$, by dimensional parity [1, Chapter V, 3.2]. Thus $G=\mathrm{SO}(3)$ or $\mathrm{Sp}(1)$ effectively and $k=3$, $\operatorname{dim}_{Z_{2}} F(G, M)=n-5$ and $\operatorname{dim}_{z} F\left(T_{0}, M\right)=n-4$, since it is of the same parity as $n$ and is at most $n-k-1=n-4$ by Lemma 2.1 . We will sometimes denote $F(G, M)$ by $F$.

Lemma 4.1. $E=\varnothing$ near $x$.

Proof. As in the proof of $[1$, Chapter XV, 6.1], we see that if there were exceptional orbits arbitrarily near $x$, then there would be a $p$-Sylow subgroup $P \subset G_{z}$ for some $z \in E$ such that ord $P \nmid$ ord $H$, and, noticing that $P \subset N\left(T_{0}\right)$ for some $T_{0}$, such that $\operatorname{dim}_{z_{p}} F(P, M) \geqq \operatorname{dim}_{z_{p}} F\left(N\left(T_{0}\right), F\left(T_{0}, M\right)\right)+1 \geqq n-4$ (since $F\left(N\left(T_{0}\right), F\left(T_{0}, M\right)\right)$ is either the $(n-4)-\mathrm{cm}_{z} F\left(T_{0}, M\right)$ or is an $\left.(n-5)-\mathrm{cm}_{Z}\right)$, and furthermore if $P \subset T_{0}$ for some $T_{0}$, then it may be assumed that $\operatorname{dim}_{z_{p}} F(P, M) \geqq \operatorname{dim}_{z_{p}} F\left(T_{0}, M\right)+1=n-3$.

Now, if $P \nsubseteq T_{0}$, then $N(P)$ is finite and it follows easily that $\operatorname{dim}_{z_{p}} E^{*}$ $\geqq \operatorname{dim}_{z_{p}} F(P, M) \geqq n-4$, and therefore $\operatorname{dim}_{z_{p}} E \geqq \operatorname{dim}_{z_{p}} E^{*}+3 \geqq n-1$, contrary to a theorem of Montgomery and Yang [5, Theorem 4].

Similarly if $P \subset T_{0}$ and $P \neq$ center $G$, then $\operatorname{dim}_{z_{p}} E^{*} \geqq \operatorname{dim}_{z_{p}} F(P, M)-1$ $\geqq n-4$, which implies that $\operatorname{dim}_{z_{p}} E \geqq n-4+3$ which is impossible. On the other hand if $P=$ center $G(G=\mathrm{Sp}(1))$, then $\operatorname{dim}_{z_{p}} F(P, M)=n-2$ by dimensional parity. Consider the action of $G / P \approx \mathrm{SO}(3)$ on the $(n-2)-\mathrm{cm}_{z_{2}} F(P, M)$. Since $F(P, M)$ is assumed to contain points in $E$, the maximum dimension of the orbits of this action is three, but since $\operatorname{dim}_{z_{2}} F(G, M)=n-5$ and $F(G / P, F(P, M))=F(G, M) \approx(F(G, M)) *$ this is seen to contradict Corollary 6.3 .

LEMMA 4.2. $N\left(T_{0}\right)$ acts trivially on $F\left(T_{0}, M\right)$.

Proof. Assume the contrary. Then $N\left(T_{0}\right) / T_{0} \approx Z_{2}$ acts effectively on $F\left(T_{0}, M\right)$ and hence $F\left(N\left(T_{0}\right), M\right)$ is an $(n-5)-\mathrm{cm}_{z}$ by the CDT $(k=0)$ (or by [7, Corollary to Lemma 16]). Thus it coincides with $F(G, M)$ near some point $y \in F(G, M)$ arbitrarily near $x$. Near $y$ there are exactly three orbit 
types: fixed points; $G / T_{0} \approx S^{2}$; and $G / H$. Note also that, near $y, B^{*}$ $\approx F\left(T_{0}, M\right) / N\left(T_{0}\right)$ is an $(n-4)-\mathrm{cm}_{z}$ with boundary $F(G, M)$ so that $H_{c}^{i}\left(B^{*}, Z\right)={ }_{L F} 0$ for all $i$ and

$$
H_{c}^{i}\left(B^{*}-F, Z\right)={ }_{L F} \begin{cases}Z, & i=n-4 \\ 0, & \text { otherwise }\end{cases}
$$

near $y$. We shall restrict the discussion in the rest of this proof to small neighborhoods of $y$.

Note also that $F\left(N\left(T_{0}\right), M\right)$ separates $F\left(T_{0}, M\right)$ into two subsets, near $y$, each of which is a cross-section for $B-F \rightarrow B^{*}-F$. Thus $B-F \approx\left(B^{*}-F\right)$ $\times S^{2}$.

Consider the Leray spectral sequence of the map $B \rightarrow B^{*}$. We see that

Thus

$$
E_{2}^{p, q}={ }_{L F} \begin{cases}Z, & p=n-4 \text { and } q=2 \\ 0, & \text { otherwise. }\end{cases}
$$

$$
H_{c}^{i}(B, Z)={ }_{L F} \begin{cases}Z, & i=n-2, \\ 0, & \text { otherwise }\end{cases}
$$

(and, in fact, this would imply that $B$ is an $(n-2)$-cm over $Z$ ).

The cohomology sequence of $M \bmod B$, then yields that

$$
H_{c}^{i}(M-B, Z)={ }_{L F} \begin{cases}Z, & i=n, n-1, \\ 0, & \text { otherwise }\end{cases}
$$

and hence also $H_{c}^{i}\left(M-B, Z_{2}\right)={ }_{L F} 0$ for $i \leqq n-2$. Now consider the fibre map

$$
M-B \stackrel{G / H}{\longrightarrow} \frac{(M-B)}{G} \text {. }
$$

Since $H \subset T_{0}$, it is cyclic and hence

$$
H^{1}\left(G / H, Z_{2}\right)=H^{2}\left(G / H, Z_{2}\right)= \begin{cases}0, & G=\operatorname{Sp}(1), \operatorname{ord}(H) \text { odd } \\ Z_{2}, & \text { otherwise, }\end{cases}
$$

and it follows that the structural group of $M-B \rightarrow(M-B) / G$ acts trivially on $H^{*}\left(G / H, Z_{2}\right)$. By Theorem 6.6 , this implies that $H_{c}^{f}\left((M-B) / G, Z_{2}\right)={ }_{L y} 0$ for $i \leqq n-2$. However $(M-B) / G$ is an $(n-3)$-cm over $Z$ and hence over $Z_{2}$, so that $H_{c}^{n-3}\left((M-B) / G, Z_{2}\right)={ }_{L_{y}} Z_{2}$. This contradiction finishes the proof of the lemma.

REMARK. Note that in deriving the local groups of $B$ above, we have essentially proved a special case of the converse of the CDT. (That is, if $X$ is an $(n-k)-\mathrm{cm}_{z}$ with boundary $F$ and $G / H$ is a cohomology $k$-sphere, then $F \cup((X-F) \times G / H)$ is an $n$-cmz.) That the converse of the CDT is true in general may be seen in exactly the same manner. 
It follows from Lemma 4.2 and the CDT that $N\left(T_{0}\right) / H \approx S^{1}$. If $G=\operatorname{Sp}(1)$, then this implies that $H$ contains the center of $G$, and hence that center $(G)$ acts trivially on $M$. Thus $G \approx \mathrm{SO}(3)$ effectively. Note that we now have that near $x$ there are exactly three types of orbits: fixed points; $G / N\left(T_{0}\right) \approx P^{2}$; and $G / H$.

LEMmA 4.3. $H \approx Z_{2} \oplus Z_{2}$.

Proof. Let $Z_{2} \oplus Z_{2} \approx K \subset N\left(T_{0}\right)$. We first show that a conjugate of $K$ is contained in $H$. Note that $F\left(K, G / N\left(T_{0}\right)\right)$ consists of three points, so that if $K$ is not conjugate to a subgroup of $H$, then $\operatorname{dim}_{Z_{2}} F(K, M)=\operatorname{dim}_{Z_{2}}(F(K, M))$ * $=\operatorname{dim}_{Z_{2}} B^{*}=n-4$. Since the three subgroups of $K$ isomorphic to $Z_{2}$ are conjugate in $G$, their fixed point sets have the same dimension, $j$, over $Z_{2}$. Borel's formula [1, Chapter XIII, 4.3] then reads

$$
4=n-(n-4)=3(j-(n-4))
$$

and this contradiction shows that $K \subset H$ (up to conjugacy).

Thus $H$ is dihedral of order $4 a, a \geqq 1$. Say that $a>1$, and note that in that case $F(H, G / H)=N(H) / H \approx Z_{2}$ consists of two points and, in fact, these points are in $F\left(H, N\left(T_{0}\right) / H\right)$. Using the CDT we easily see from this that $F(H, M-F(G, M))$ is an $(n-3)$-cm over $Z$. But $F(H, M-F) \subset F(K, M-F)$ which is a connected $(n-3)$-cm over $Z_{2}$. Since $F(H, M-F)$ is closed in $F(K, M-F)$ this implies that $F(H, M-F)=F(K, M-F)$ and this is inconsistent with the fact that $F\left(K, G / N\left(T_{0}\right)\right)$ consists of three points while $F\left(H, G / N\left(T_{0}\right)\right)$ is one point. Thus $a=1$ and $H=K \approx Z_{2} \oplus Z_{2}$ as was to be shown.

Let $X=F(H, M)$. Then $X$ is an $(n-3)$-cm over $Z_{2}$. We may assume, by taking $M$ smaller (about $x$ ), that $M$ contains no exceptional orbits and hence that the isotropy groups in $M$ are conjugates of $G, N\left(T_{0}\right)$, and $H$.

Put $B^{\prime}=B \cap X . X-B^{\prime}$ is then a principal $N(H) / H$-bundle over $(M-B) / G$ $\approx\left(X-B^{\prime}\right) / N(H)$, and $N(H) / H \approx \sigma_{3}$, the group of permutations of three objects. We wish to show that this bundle is trivial if $M$ is sufficiently small. Let $J \approx Z_{3}$ be the subgroup of $N(H) / H \approx \sigma_{3}$ of order 3 . We then have the bundle maps

$$
X-B^{\prime} \stackrel{Z_{3}}{\longrightarrow} \frac{X-B^{\prime}}{J} \stackrel{Z_{2}}{\longrightarrow} \frac{X-B^{\prime}}{N(H)} \approx \frac{M-B}{G} .
$$

Since $X$ is an $(n-3)-\mathrm{cm}_{Z_{2}}$ it follows easily from [1, Chapter III, 2.3] that $X / J$ is also an $(n-3)-\mathrm{cm}_{z_{2}}$. Since $F((N(H) / H) / J, X / J)=B^{\prime} / J$ is $(n-4)$-dimensional we have that the second bundle map above is trivial (near $x$ ) by [7, Lemma 16]. (Note that it also follows that $X / N(H) \approx M / G$ is an $(n-3)-\mathrm{cm}_{Z_{2}}$ with boundary $B^{\prime} / N(H) \approx B / G$.) Thus $X-B^{\prime}$ breaks into two parts each of which is a $J$-bundle over $(M-B) / G$.

Note that, since $G / N\left(T_{0}\right) \approx P^{2}$ we have that $\pi^{*}: H_{c}^{i}\left(B^{*}, Z_{3}\right) \rightarrow H_{c}^{i}\left(B, Z_{3}\right)$ is 
an isomorphism (onto) and, since $B^{*} \approx F\left(T_{0}, M\right)$ is an $(n-4)-\mathrm{cm}_{z}$, we have that $H_{c}^{i}\left(B, Z_{3}\right)=0$ for $i \geqq n-3$. This implies that $H_{c}^{n-1}\left(M-B, Z_{3}\right)={ }_{L x} 0$. Thus for the spectral sequence $E_{r}^{p, q}$, with coefficients in $Z_{3}$, of the fibering $M-B$ $\rightarrow(M-B) / G$ we have (see $[1$, Chapter $\mathrm{XV}, 6.2]$ )

$$
0={ }_{L x} H_{c}^{n-1}\left(M-B, Z_{3}\right) \approx E_{\infty}^{n-4,3} \approx E_{2}^{n-4,3} \approx H_{c}^{n-4}\left(\frac{M-B}{G}, Z_{3}\right),
$$

since $H^{*}\left(G / H, Z_{3}\right)=H^{*}\left(S^{3}, Z_{3}\right)$. Also note that

$$
H_{c}^{n-3}\left(\frac{M-B}{G}, Z_{3}\right) \approx E_{2}^{n-3,3} \approx E_{\infty}^{n-3,3} \approx H_{c}^{n}\left(M-B, Z_{3}\right) \approx Z_{3}
$$

so that $(M-B) / G$ is orientable. Thus, by Theorem 6.5 , we have that (near $x$ ) $X-B^{\prime}$ splits into six parts each homeomorphic via $\pi$ to $(M-B) / G$.

LEMMA 4.4. $H_{c}^{i}\left(F, Z_{2}\right)={ }_{L F} 0$ for $i \leqq n-6$, and $H_{c}^{i}\left((B-F) / G, Z_{2}\right)={ }_{L F} 0$ for $i \leqq n-5$.

Proof. It has been shown above that $M / G$ is an $(n-3)$-cm over $Z_{2}$ with boundary $B / G$ and that there is a cross-section in $F(H, M)$ for the action of $G$ on $M-B$. Thus $H_{c}^{i}\left((M-B) / G, Z_{2}\right)={ }_{L_{y}} 0$ for $i \leqq n-4$ and any $y \in F(G, M)$. Also $M-B \approx(M-B) / G \times G / H$ so that $H_{c}^{i}\left(M-B, Z_{2}\right)={ }_{L y} 0$ for $i \leqq n-4$.

Since $X-B^{\prime}$ is the union of six disjoint copies of $(M-B) / G$ we have $H_{c}^{\prime}\left(X-B^{\prime}, Z_{2}\right)={ }_{L y} 0$ for $i \leqq n-4$. Since $X$ is an $(n-3)-\mathrm{cm}_{z_{2}}$ this implies that $H_{c}^{i}\left(B^{\prime}, Z_{2}\right)={ }_{L y} 0$ for $i \leqq n-5$.

Note that if $T_{0}, T_{1}$, and $T_{2}$ are the three circle subgroups of $G$ with normalizers containing $H$, then, since $F\left(T_{i}, M\right)=F\left(N\left(T_{i}\right), M\right) \subset F(H, M)$ $=X$, we have $F\left(T_{0}, M\right) \cup F\left(T_{1}, M\right) \cup F\left(T_{2}, M\right)=B^{\prime}$ and $B^{\prime}-F$ is the disjoint union of the $\left(F\left(T_{i}, M\right)-F\right)$. Also $\pi$ is a homeomorphism on each $\left(F\left(T_{i}, M\right)-F\right)$ to $(B-F) / G$. It follows that $\pi^{*}: H_{c}^{i}\left((B-F) / G, Z_{2}\right) \rightarrow H_{c}^{i}\left(B^{\prime}-F\right)$ cannot be surjective unless these groups are trivial and this also holds in the local sense. Thus, by the diagram (coefficients in $Z_{2}$ )

$$
\begin{aligned}
& H_{c}^{i-1}(B / G) \rightarrow H_{c}^{i-1}(F) \rightarrow H_{c}^{i}\left(\frac{B-F}{G}\right) \rightarrow H_{c}^{i}(B / G) \\
& \underset{\downarrow}{\downarrow} \approx \underset{H_{c}^{i-1}(F) \rightarrow H_{c}^{i}\left(B^{\prime}-F\right)}{\downarrow} \rightarrow \underset{H_{c}^{i}\left(B^{\prime}\right)}{\longrightarrow}
\end{aligned}
$$

and the fact, shown above, that $H_{c}^{i}\left(B^{\prime}, Z_{2}\right)={ }_{L y} 0$ for $i \leqq n-5$, it follows easily that $H_{c}^{t}\left((B-F) / G, Z_{2}\right)={ }_{L y} 0$ for $i \leqq n-5$.

Since $B / G \approx F\left(T_{0}, M\right)$ is an $(n-4)$-cm over $Z$ and hence over $Z_{2}$, it also follows that $H_{c}^{i-1}\left(F, Z_{2}\right)={ }_{L y} 0$ for $i \leqq n-5$, that is, for $i-1 \leqq n-6$, as was to be shown.

We will now complete the proof of Case II. Let $y \in F=F(G, M)$ be arbi- 
trary. Note that, since $F\left(T_{0}, G / N\left(T_{0}\right)\right)$ is a point, we have that $F\left(T_{0}, B-F\right)$ $=F\left(N\left(T_{0}\right), B-F\right)$ is a cross-section for the orbits in $B-F$ so that

$$
B-F \approx \frac{B-F}{G} \times P^{2} .
$$

Consider the Leray spectral sequence $E_{r}^{p, q}$ (coefficients in $Z$ ) of the map $B \rightarrow{ }^{\pi} B / G$. We have

$$
E_{2}^{p, q}=H_{c}^{p}\left(B / G, \mathfrak{H}^{q}\left(G / G_{w}, Z\right)=0 \quad \text { for } q \neq 0,2,\right.
$$

since $G / G_{w}$ is a point for $w \in F$, and is a projective plane for $w \in B-F$. Since the sheaf $\operatorname{FC}^{2}\left(G / G_{w}, Z\right)$ is zero on $F$ and constant on $(B-F) / G$ with stalks $H^{2}\left(P^{2}, Z\right) \approx Z_{2}$, we have: $E_{2}^{p, q}=0, q \neq 0,2 ; E_{2}^{p, 0} \approx H_{c}^{p}(B / G, Z)=_{L y} 0$, for $p \neq n-4$; and $E_{2}^{p, 2} \approx H_{c}^{p}\left((B-F) / G, Z_{2}\right)={ }_{L y} 0$, for $p \neq n-4$. It follows that $H_{c}^{i}(B, Z)={ }_{L y} 0$, for $i \neq n-4, n-2$. Thus also $H_{c}^{i}(M-B, Z)={ }_{L y} 0$, for $i \leqq n-4$, and since $M-B \approx(M-B) / G \times G / H$, this implies that $H_{c}^{i}((M-B) / G, Z)$ $={ }_{L y} 0$, for all $i \leqq n-4$.

Since $(M-B) / G$ is an orientable $(n-3)$-cm over $Z$ and is connected (even locally near $y$ ) because $M-B$ is connected, and since $M / G-F$ is an $(n-3)-\mathrm{cm}_{z}$ with boundary $B / G-F$ by the CDT, it follows from Lemma 2.6 that $M / G$ is an $(n-3)-\mathrm{cm}_{z}$ with boundary $B / G$, which completes the proof of Case II.

We shall now show that $F$ is an $(n-5)$-cm over $Z_{2}$, since this will be needed in the treatment of Case III.

By the fact that $M-B \approx(M-B) / G \times G / H$ and that $H^{1}\left(G / H, Z_{2}\right)$ $\approx H^{2}\left(G / H, Z_{2}\right) \approx Z_{2} \oplus Z_{2}$, we have that $H_{c}^{n-2}\left(M-B, Z_{2}\right)={ }_{L F} Z_{2} \oplus Z_{2}$ and hence that $H_{c}^{n-3}\left(B, Z_{2}\right)={ }_{L F} Z_{2} \oplus Z_{2}$. We again consider the Leray spectral sequence of $B \rightarrow B / G$, but with coefficients in $Z_{2}$. We have that $E_{2}^{p, q}={ }_{L F} 0$ for $q>2$ or for $p \neq n-4$. It follows readily that

$$
H_{c}^{n-4}\left(\frac{B-F}{G}, Z_{2}\right) \approx E_{2}^{n-4,1}={ }_{L F} E_{\infty}^{n-4,1}={ }_{L F} H_{c}^{n-3}\left(B, Z_{2}\right)={ }_{L F} Z_{2} \oplus Z_{2} .
$$

Now the cohomology sequence

$$
H_{c}^{n-5}\left(B / G, Z_{2}\right) \rightarrow H_{c}^{n-5}\left(F, Z_{2}\right) \rightarrow H_{c}^{n-4}\left(\frac{B-F}{G}, Z_{2}\right) \rightarrow H_{c}^{n-4}\left(B / G, Z_{2}\right) \rightarrow 0
$$

is locally about $F$

$$
0 \rightarrow H_{c}^{n-5}\left(F, Z_{2}\right) \rightarrow Z_{2} \oplus Z_{2} \rightarrow Z_{2} \rightarrow 0
$$

and it can be seen easily that this implies that $H_{c}^{n-5}\left(F, Z_{2}\right)={ }_{L F} Z_{2}$ and our contention follows from Lemma 4.4 (see [1, Chapter XV, 2.2]). 
5. Case III. $\operatorname{rank}(G)=\operatorname{rank}(H)+2$. It will suffice to treat the case in which $H$ is finite and $G$ is connected. Hence $\operatorname{rank}(G)=2$. Note that by Lemma 2.1, $\operatorname{dim}_{z} F\left(T_{0}, M\right)=n-k-2$ so that $F(G, M)$ and $F\left(T_{0}, M\right)$ coincide in some open set near $x$ (but not necessarily containing $x$ ). It follows that $\operatorname{dim}_{L}(F(G, M), x)=n-k-2$ over any coefficient ring $L$. We first prove a lemma useful for the treatment of $E$.

Lemma 5.1. Let $P$ be a p-group with $\operatorname{dim} N(P)>0$; then either $P$ is conjugate to a subgroup of $H$ or $F(P, M) \cap E=\varnothing$ near $x$.

Proof. Assume that $P$ is not conjugate to a subgroup of $H$ but that $F(P, M) \cap E \neq \varnothing$ near $x$, that is, the component of the $\mathrm{cm}_{z_{p}} F(P, M)$ containing $x$ also contains points of $E$. Let $z$ be such a point. For convenience of notation we shall assume that $F(P, M)$ is connected. We may assume that if $\boldsymbol{z}^{\prime} \in F(P, M) \cap E$ is sufficiently close to $z$, then $G_{z} \sim G_{z^{\prime}}$. Now $F\left(P, G / G_{z}\right)$ is easily seen to have the same dimension as $N(P)$ (since $G_{z}$ is a finite group), and moreover, near $z$, the map $F(P, M) / N(P) \rightarrow F(P, M)^{*}$ is finite to one and hence cannot lower dimension. Thus

$$
\operatorname{dim}_{Z_{p}} \frac{F(P, M)}{N(P)} \leqq \operatorname{dim}_{Z_{p}}\left(F(P, M)^{*}, z^{*}\right) \leqq \operatorname{dim}_{z_{p}}\left(E^{*}, z^{*}\right) \leqq n-k-2,
$$

since if it were $\geqq n-k-1$, then $\operatorname{dim}_{Z_{p}} E \geqq n-1$ contrary to [5, Theorem 4]. But $F(G, M) \approx F(G, M) / N(P)$ is contained in the singular set of the action of $N(P)$ on $F(P, M)$ so that by Corollary $6.3, \operatorname{dim}_{z_{p}} F(G, M) \leqq n-k-3$ (even near $x)$, which contradicts the fact that $\operatorname{dim}_{L}(F(G, M), x)=n-k-2$.

The following lemma is the main tool of this section.

Lемма 5.2. $G$ is not a simple group. Furthermore, there are exactly two mutually nonconjugate circle groups $S_{1}$ and $S_{2}$ contained in $T_{0}$ such that, for any circle group $S \subset T_{0}$, we have that $\operatorname{dim}_{Z}(F(S, M), x)>\operatorname{dim}_{Z}\left(F\left(T_{0}, M\right), x\right)$ $=n-k-2$ if and only if $S$ is conjugate to one of the $S_{i}, i=1,2$.

Proof. Any rank two group is locally isomorphic to one of the following groups: $D_{1} \times D_{1} ; D_{1} \times A_{1} ; A_{1} \times A_{1} ; A_{2} ; B_{2} ;$ or $G_{2}$ (where $D_{1}$ denotes the circle group $\mathrm{SO}(2)$ and the other notation is standard). These have dimensions $2,4,6,8,10$, and 14 respectively. The corresponding Weyl groups $W(G)$ are $(e), D(2) \approx Z_{2}, D(4) \approx Z_{2} \oplus Z_{2}, D(6), D(8)$, and $D(12)$, where $D(m)$ denotes the dihedral group of order $m$.

If $S \subset T_{0}$ is a circle group, then let $n(S)$ be the number of conjugates of $S$ which are contained in $T_{0}$. As may be seen by consideration of the action of $W(G)$ on $\tilde{T}_{0} \approx E^{2}$, the situation is as shown in Table 1 .

Since in Lemma 2.1 we have equality for the first two inequalities, it follows from the remark below 2.1 that for any $S$ with $\operatorname{dim}(F(S, M), x)$ $>n-k-2$ we have 
TABLE 1

\begin{tabular}{|c|c|c|c|}
\hline$G$ & $S$ & $n(S)$ & $\begin{array}{c}\text { Number of nonconjugate } \\
S \subset T_{0} \text { having this } \\
\text { value of } n(S)\end{array}$ \\
\hline$D_{1} \times D_{1}$ & $(-)$ & 1 & $\infty$ \\
\hline \multirow{3}{*}{$D_{1} \times A_{1}$} & singular & 1 & 1 \\
\hline & \multirow{2}{*}{ regular } & 1 & 1 \\
\hline & & 2 & $\infty$ \\
\hline \multirow{2}{*}{$A_{1} \times A_{1}$} & singular & 1 & 2 \\
\hline & regular & 2 & $\infty$ \\
\hline \multirow{3}{*}{$A_{2}$} & singular & 3 & 1 \\
\hline & \multirow{2}{*}{ regular } & 3 & 1 \\
\hline & & 6 & $\infty$ \\
\hline \multirow{2}{*}{$B_{2}$} & singular & 2 & 2 \\
\hline & regular & 4 & $\infty$ \\
\hline \multirow{2}{*}{$G_{2}$} & singular & 3 & 2 \\
\hline & regular & 6 & $\infty$ \\
\hline
\end{tabular}

[Also note that, by the proof of Lemma 2.1, we have that for such an $S \subset T_{0}$, $\operatorname{dim}_{Z} F(S, M) *=n-k-1$.]

Let $p_{i}$ be the number of mutually nonconjugate $S \subset T_{0}$ such that $\operatorname{dim}_{Z}(F(S, M), x)>n-k-2$, for $S$ singular and $n(S)=i$. Similarly, let $q_{i}$ be the analogous number for $S$ regular. Then the formula of Borel [1, Chapter XIII, 4.3] implies that

$$
k+2=n-(n-k-2)=\sum_{i}\left(4 i p_{i}\right)+\sum_{i}\left(2 i q_{i}\right) .
$$

With the restrictions imposed by Table 1 this formula is as in Table 2 (in which any $p_{i}$ or $q_{i}$ is zero if it does not appear explicitly).

We see immediately that the formula cannot be solved in the last three cases, so that $G$ is not simple, and in the first three cases we must have $\sum p_{i}$ $+\sum q_{i}=2$ which is exactly the remainder of the statement of Lemma 5.2. 
TABLE 2

\begin{tabular}{c|c}
\hline \hline$G$ & Formula of Borel \\
\hline$D_{1} \times D_{1}$ & $4=2 q_{1}$. \\
\hline$D_{1} \times A_{1}$ & $6=4 p_{1}+2 q_{1}+2 \cdot 2 q_{2} ; p_{1}=0,1 ; q_{1}=0,1$. \\
\hline$A_{1} \times A_{1}$ & $8=4 p_{1}+2 \cdot 2 q_{2} ; p_{1}=0,1,2$. \\
\hline$A_{2}$ & $10=4 \cdot 3 p_{3}+2 \cdot 3 q_{3}+2 \cdot 6 q_{6} ; p_{3}=0,1 ; q_{3}=0,1$. \\
\hline$B_{2}$ & $12=4 \cdot 2 p_{2}+2 \cdot 4 q_{4} ; p_{2}=0,1,2$. \\
\hline$G_{2}$ & $16=4 \cdot 3 p_{3}+2 \cdot 6 q_{6} ; p_{3}=0,1,2$. \\
\hline
\end{tabular}

REMARK. Note that in case $G$ is locally isomorphic to $D_{1} \times A_{1}$, we must have $q_{1}=1$, which means that one of the $S_{i}$ is contained in the 3-dimensional factor $A_{1}$.

Now consider the action of $N\left(S_{i}\right)$ on $F\left(S_{i}, M\right)$ (near $\left.x\right)$. As can be seen from the proof of Lemma 2.1, $\operatorname{dim}_{z} F\left(S_{i}, M\right) / N\left(S_{i}\right)=n-k-1$ and hence the action of $N\left(S_{i}\right)$ on $F\left(S_{i}, M\right)$ satisfies the hypotheses of the CDT. Hence $N\left(S_{i}\right)$ has exactly two types of orbits near $x$ on $F\left(S_{i}, M\right)$, principal orbits and fixed points and the fixed point set $F\left(N\left(S_{i}\right), M\right)$ must be an $(n-k-2)-\mathrm{cm}_{z}$. Since $F\left(T_{0}, M\right) \subset F\left(N\left(S_{i}\right), M\right)$ is an $(n-k-2)-\mathrm{cm}_{z}$ we have that $F\left(T_{0}, M\right)$ $=F\left(N\left(S_{i}\right), M\right)$, near $x$, and its complement in $F\left(S_{i}, M\right)$ is the set of principal orbits of $N\left(S_{i}\right)$ on $F\left(S_{i}, M\right)$. It follows that $F\left(S_{i}, M\right)-F\left(T_{0}, M\right)$ consists of points with isotropy groups (in $G$ ) of rank one (and hence with $S_{i}$ as a maximal torus). Also by the CDT we must have that the principal orbits of $N\left(S_{i}\right)$ on $F\left(S_{i}, M\right)$ are cohomology spheres (of odd dimension). Thus if $x_{i} \in F\left(S_{i}, M\right)$ $-F\left(T_{0}, M\right)$ and $K_{i}=G_{x_{i}}$ then

$$
\frac{N\left(S_{i}\right)}{N\left(S_{i}\right) \cap K_{i}} \text { is an integral cohomology sphere. }
$$

Now choose $x_{i} \in F\left(S_{i}, M\right)-F\left(T_{0}, M\right)$ in such a way that the $K_{i}$ are locally minimal isotropy groups for points in $F\left(S_{i}, M\right)$ (that is, if $x_{i}^{\prime} \in F\left(S_{i}, M\right)$ is sufficiently close to $x_{i}$ then $\left.G_{x_{i}^{\prime}} \sim G_{x_{i}}=K_{i}\right)$. Some conjugate of $H$, say $H_{i}$, is a principal isotropy group of the action of $K_{i}$ on a slice at $x_{i}$. Note that this action of $K_{i}$ on a slice at $x_{i}$ satisfies the hypotheses of the CDT so that

$$
\frac{K_{i}}{H_{i}} \text { is an integral cohomology sphere. }
$$

Using these facts we shall attempt to classify the groups $\left(G, K_{1}, K_{2}, H\right)$. We will obtain, as the only possibilities, the cases in Table 3 . ( $I$ denotes an icosahedral subgroup of $\mathrm{SO}(3)$ and $I^{\prime}$ denotes an icosahedral subgroup of $\mathrm{Sp}(1)$.) 
TABLE 3

\begin{tabular}{|c|c|c|c|c|}
\hline Case & $G$ & $K_{1}$ & $K_{2}$ & $H$ \\
\hline 1 & $\mathrm{SO}(2) \times \mathrm{SO}(2)$ & $\mathrm{SO}(2) \times(e)$ & $(e) \times \mathrm{SO}(2)$ & $(e)$ \\
\hline 2 & $\mathrm{SO}(3) \times \mathrm{SO}(2)$ & $\mathrm{SO}(3) \times(e)$ & $I \times S O(2)$ & $I \times(e)$ \\
\hline 3 & $\mathrm{SO}(3) \times \mathrm{SO}(2)$ & $\begin{array}{l}S_{1} H\left(S_{1} \subset \mathrm{SO}(3)\right) \\
\left(K_{1} \text { not abelian }\right)\end{array}$ & $\begin{array}{l}S_{2} \text { (regular) } \\
\left(K_{2} \cap \operatorname{SO}(3)=(e)\right)\end{array}$ & $\begin{array}{l}Z_{2} \\
(H \cap \operatorname{SO}(3)=(e))\end{array}$ \\
\hline 4 & $\mathrm{Sp}(1) \times \mathrm{SO}(2)$ & $\mathrm{Sp}(1) \times(e)$ & $(e) \times \mathrm{SO}(2)$ & $(e)$ \\
\hline 5 & $\mathrm{Sp}(1) \times \mathrm{SO}(2)$ & $\mathrm{Sp}(1) H$ & $\begin{array}{l}S_{2} \text { (regular) } \\
\left(K_{2} \cap \operatorname{Sp}(1)=(e)\right)\end{array}$ & $\begin{array}{l}\text { cyclic } \\
(H \cap \mathrm{Sp}(1)=(e))\end{array}$ \\
\hline 6 & $\mathrm{SO}(3) \times \mathrm{SO}(3)$ & $\mathrm{SO}(3) \times I$ & $I \times \mathrm{SO}(3)$ & $I \times I$ \\
\hline 7 & $\mathrm{SO}(3) \times \mathrm{Sp}(1)$ & $\mathrm{SO}(3) \times(e)$ & $I \times \mathrm{Sp}(1)$ & $I \times(e)$ \\
\hline 8 & $\mathrm{Sp}(1) \times \mathrm{Sp}(1)$ & $\mathrm{Sp}(1) \times(e)$ & $(e) \times \operatorname{Sp}(1)$ & $(e)$ \\
\hline 9 & $\mathrm{Sp}(1) \times \mathrm{Sp}(1)$ & $\mathrm{Sp}(1) \times(e)$ & $\mathrm{Sp}(1)$ (diagonal) & $(e)$ \\
\hline 10 & $\frac{\mathrm{Sp}(1) \times \mathrm{Sp}(1)}{Z_{2}}$ & $\frac{\mathrm{Sp}(1) \times I^{\prime}}{Z_{2}}$ & $\mathrm{SO}(3)$ (diagonal) & $I\left(\subset K_{2}\right)$ \\
\hline 11 & $\frac{\mathrm{Sp}(1) \times \mathrm{Sp}(1)}{Z_{2}}$ & $S_{1} H \approx \mathrm{O}(2)$ & $S_{2} H \approx \mathrm{O}(2)$ & $\begin{array}{l}Z_{2}+Z_{2} \\
\text { (HCSO(3), the } \\
\text { "diagonal") }\end{array}$ \\
\hline
\end{tabular}

In all cases in the table except (5) the action is assumed to be effective, while in case (5), for convenience, we assume only that $G$ is almost effective, so that the effective group is $G /(H \cap$ center $G)$. In cases (10) and (11) the group $G$ is $\mathrm{Sp}(1) \times \mathrm{Sp}(1)$ divided by the "diagonal" central element, that is $G \approx \mathrm{SO}(4)$.

REMARK. Examples can be given for all the cases (1) through (10). For cases (1), (3), (4), (5), (8) and (9) linear examples can be constructed and this can be seen to be impossible in the other cases. We strongly suspect that case (11) cannot arise, but we know of no way to see this. The exact situation in cases (3) and (5) is also in doubt.

For the proof of the "classification" we shall assume, unless otherwise specified, that $G=G_{1} \times G_{2}, G_{i} \approx \mathrm{SO}(2)$, or $\mathrm{Sp}(1)$ and that $G$ is only almost effective, so that the effective group is obtained by dividing out by

$$
H \cap \text { center }(G) \text {. }
$$


Note that $\mathrm{Sp}(1) / I^{\prime} \approx \mathrm{SO}(3) / I$ is an integral cohomology 3 -sphere. If $G_{i} \approx \mathrm{SO}(2)$ then we can (and shall) assume, without loss of generality, that $H \cap G_{i}=(e)$.

In case $G_{i}=\mathrm{SO}(2), i=1,2, H$ may be assumed to be trivial, since it is central. Thus $K_{i} / H_{i}=K_{i}$, to be a cohomology sphere, must be $S_{i}$. It remains to show that $S_{1} \cap S_{2}=(e)$. If not then let $P \subset S_{1} \cap S_{2}, P \approx Z_{p}$ for some prime $p$. Then $F(P, M)$ is a $\mathrm{cm}_{Z_{p}}$ properly containing the $(n-2)-\mathrm{cm}_{Z} F\left(S_{1}, M\right)$. Thus $\operatorname{dim}_{z_{p}} F(P, M)=n-1$, which is impossible since $P$ preserves orientation (see $[1$, Chapter V, 2.3]).

Now in the other cases, if $S_{i} \subset G_{i}, i=1,2$, then every conjugate of $S_{1}$ commutes with every conjugate of $S_{2}$. From this we see easily that it may be assumed in this case that $H_{1}=H=H_{2}$. We also see that this can be assumed if either $K_{i}$ contains one of the $G_{i}$, since such a $K_{i}$ must intersect every maximal torus of $G$. (That is, if $K_{1} \supset G_{1}$ say, then we could replace $K_{1}$ by any conjugate $g K_{1} g^{-1}$ and still have $g K_{1} g^{-1} \cap T_{0} \supset S_{1}$. Thus $g$ could be chosen so that $H_{2}$ is a principal isotropy group of the action of $g K_{1} g^{-1}=g G_{x_{1}} g^{-1}=G_{g\left(x_{1}\right)}$ on a slice at $\left.g\left(x_{1}\right).\right)$

Say that $G=G_{1} \times G_{2}, G_{1} \approx \mathrm{Sp}(1)$ and $G_{2} \approx \mathrm{SO}(2)$. First assume that $S_{1} \subset G_{1}$, $S_{2} \subset G_{2}$. Then, since $G / K_{2}=N\left(S_{2}\right) /\left(N\left(S_{2}\right) \cap K_{2}\right)$ is a cohomology sphere, we see that $K_{2}=S_{2}$ or $K_{2}=I^{\prime} \times S_{2}$. Thus $H=(e)$ or $H=I^{\prime} \times(e)$ respectively. If $H=(e)$ then, since $K_{1} / H$ is a cohomology sphere, we have $K_{1}=S_{1}$ or $K_{1}=G_{1}$. But, if $K_{1}=S_{1}$, then $N\left(S_{1}\right) /\left(N\left(S_{1}\right) \cap K_{1}\right)$ is not connected. Thus $K_{1}=G_{1}$ and this yields case (4). If $H=I^{\prime} \times(e)$, then clearly $K_{1}=G_{1}$ in order for $K_{1} / H$ to be a cohomology sphere. This yields case (2).

Now say that $S_{1} \subset G_{1}, S_{2}$ is regular, and $\operatorname{dim} K_{1}=1$. First assume that $G_{1} \approx \mathrm{SO}(3)$ effectively so that we may assume that $G \approx \mathrm{SO}(3) \times \mathrm{SO}(2)$ is effective. We have that $N\left(S_{2}\right)=T_{0}$ and, since $K_{2}^{0}=S_{2}$, we have $H_{2} \subset K_{2} \subset N\left(S_{2}\right)$ $=T_{0}$. Also $N\left(S_{1}\right)=N_{G_{1}}\left(S_{1}\right) \times G_{2}=N\left(T_{0}\right)$ and, since

$$
\frac{N\left(S_{1}\right)}{N\left(S_{1}\right) \cap K_{1}}=\frac{N\left(S_{1}\right)}{K_{1}}
$$

must be a circle, we have that $K_{1}$ contains an element of the form $g=g_{1} g_{2}$, where $g_{1} \in N_{G_{1}}\left(S_{1}\right)-S_{1}$ and $g_{2} \in G_{2}$. Since $K_{1} / H_{1}$ is also a circle, $H_{1}$ must contain an element of the coset $g S_{1}$ and this element may be assumed to be $g$. Then, since $H_{1}$ contains the central element $g^{2}=g_{1}^{2} g_{2}^{2}=g_{2}^{2}$, and since $G$ is effective, we have $g_{2}^{2}=(e)$. We now claim that $K_{2} \cap G_{1}=(e)$. If not there is a $p$ group $P \subset K_{2} \cap S_{1}$, for some prime $p$, and hence for which $F(P, M)$ contains the $(n-4)$-cm $F\left(S_{1}, M\right)$ properly. Then we see that $P$ must be conjugate to a subgroup of $H$, since if not we would have

$\operatorname{dim}_{Z_{p}} F(P, M)=\operatorname{dim}_{Z_{p}}\left[F\left(S_{1}, M\right)\right]^{*}+\operatorname{dim} F\left(P, G / K_{1}\right)=(n-5)+1=n-4$ and $F(P, M)$ could not contain the $(n-4)-\mathrm{cm} F\left(S_{1}, M\right)$ properly. But if $P$ is conjugate to a subgroup of $H$ (and hence $H_{1}$ ) we must have $P \approx Z_{2}$ (by the facts that $g \in H_{1}$ and $H_{1} \sim H_{2} \subset T_{0}$ ) and hence by dimensional parity 
$\operatorname{dim}_{Z_{2}} F(P, M)=n-2$. Consider the subgroup $J \approx Z_{2} \oplus Z_{2}$ of $G_{1} \approx \mathrm{SO}(3)$. Since $H_{2} \subset T_{0}$ we see that $J$ is not conjugate to a subgroup of $H$ and by Lemma 5.1 we have that $F(J, M) \subset B$. But all the subgroups of order two of $J$ are conjugate in $G$ to $P$ so that Borel's formula reads

$$
n-\operatorname{dim}_{Z_{2}} F(J, M)=3\left(\operatorname{dim}_{Z_{2}} F(J, M)-(n-2)\right)
$$

and hence $\operatorname{dim}_{z_{2}} F(J, M)=n-3$. But the map $F(J, M) / N(J) \rightarrow F(J, M)^{*}$ $C B^{*}$ is clearly a homeomorphism, and, since $\operatorname{dim} B^{*}=n-5$ and $\operatorname{dim} N(J)$ $=1$, this is a contradiction. Thus $K_{2} \cap G_{1}=(e)$ as claimed. It follows that, in fact, $H_{2} \subset S_{2}, S_{1} \cap S_{2}=(e)$, and $g_{2} \neq(e)$. It now follows immediately that $H_{2}=\{e, g\} \approx Z_{2}, K_{1}=S_{1} H_{1}$, and $K_{2}=S_{2}$. This yields case (3).

In order to treat the case in which $G_{1} \approx \mathrm{Sp}(1)$ effectively we must first investigate case (3) more fully. We see immediately from Lemma 5.1 that there are no exceptional orbits. Moreover, by Corollary 2.4 all isotropy groups of points in $F\left(S_{2}, M\right)-F\left(T_{0}, M\right)$ are conjugate to $K_{2}$. Thus if $J \approx Z_{2} \oplus Z_{2}$ is a subgroup of $G_{1}$ we see that $F(J, M) \subset B_{1}=G F\left(S_{1}, M\right)$. By Borel's formula $n-\operatorname{dim}_{z_{2}} F(J, M)$ must be divisible by 3 and hence

$$
\operatorname{dim}_{Z_{2}} F(J, M)=n-3 \text { or } n-6 .
$$

But just as above, it cannot be $n-3$ and it follows that $F(J, M)$ is precisely the $(n-6)-\mathrm{cm}_{z} F\left(N\left(T_{0}\right), M\right)$. (Note that it also follows from this that there are no points in $M$ with a 3-dimensional isotropy group.)

Now consider the case in which $G_{1} \approx \mathrm{Sp}(1)$ effectively, $G_{2} \approx \mathrm{SO}(2), S_{1} \subset G_{1}$, $S_{2}$ regular, and $\operatorname{dim} K_{1}=1$. Consider the element $e \neq g \in$ center $G_{1}$. Then $F(g, M) \supset G F\left(S_{1}, M\right)=B_{1}$ is of dimension $n-2$ (since $G_{1}$ is effective). Thus $M /(e, g)$ is an $n$-cm on which $G /(e, g)$ acts, which is case (3). Also note that $G /(e, g)$ acts on the $(n-2)-\mathrm{cm}_{z_{2}} F(g, M)$. As shown above $\operatorname{dim}_{z_{2}} F(J, M /(e, g))$ $=n-6$, and also we have shown that

$$
F(J, M /(e, g)) \subset B_{1}=G F\left(S_{1}, M /(e, g)\right) \subset \frac{F(g, M)}{(e, g)} \approx F(g, M)
$$

and hence $F(J, M /(e, g)) \approx F(J, F(g, M))$ is an $(n-6)-\mathrm{cm}_{z_{2}}$. But since $G_{1} /(e, g)$ acts on $F(g, M)$, Borel's formula implies that $(n-2)$ $-\operatorname{dim}_{Z_{2}} F(J, F(g, M))$ is divisible by 3 . This contradiction eliminates this case.

The next case to be considered is that for which $G_{1} \approx \mathrm{Sp}(1), G_{2} \approx \mathrm{SO}(2)$, $S_{1} \subset G_{1}, S_{2}$ regular and $\operatorname{dim} K_{1}=3$. Here $K_{1}^{0}=G_{1}$ and thus by the remarks above we may assume $H_{1}=H=H_{2}$. We have that $K_{1}=G_{1} \times J_{2}$ for some subgroup $J_{2} \subset G_{2}$. Since $K_{1} / H$ is an integral cohomology sphere we must have that $G_{1} \cap H=(e)$ or $I^{\prime}$. However $H \subset N\left(S_{2}\right)=T_{0}$ so that we must have $G_{1} \cap H=(e)$. We now claim that $G_{1} \cap K_{2}=(e)$. Note that since $K_{2} \subset N\left(S_{2}\right)=T_{0}$ we have $G_{1} \cap K_{2}=S_{1} \cap K_{2}$. Let $P$ be some $p$-group in $S_{1} \cap K_{2}$. Then $F(P, M)$ contains then $(n-4)-\mathrm{cm} F\left(S_{1}, M\right)$ properly. However $P$ is not conjugate 
to a subgroup of $H$ and it follows that

$$
\operatorname{dim}_{Z_{p}} F(P, M)=n-5+\operatorname{dim} F\left(P, G / K_{1}\right)=n-4,
$$

and hence $F(P, M)$ cannot contain $F\left(S_{1}, M\right)$ properly. This is a contradiction which shows that $G_{1} \cap K_{2}=(e)$ as claimed. It follows that $K_{2}=S_{2}$ and hence that $H \subset S_{2}$. This yields case (5).

We now consider the cases in which $G_{i} \approx \operatorname{Sp}(1), i=1,2, G=G_{1} \times G_{2}$ is almost effective. We first take up the case in which $S_{1} \subset G_{1}$ and $\operatorname{dim} K_{1}=1$. We claim that this situation cannot arise. To see this consider the nontrivial element $g \in$ center $G_{1}$. Since $g \in S_{1}$ we see that $F(g, M) \supset G F\left(S_{1}, M\right)$ which is of dimension $n-2$. Hence $\operatorname{dim} F(g, M)=n-2$ or $n$ and this implies that $M /(e, g)$ is an $n-\mathrm{cm}_{Z}$ on which $G /(e, g)$ acts. Thus we may assume for the present consideration that $G=G_{1} \times G_{2}$ is almost effective and $G_{1} \approx \mathrm{SO}(3)$, $G_{2} \approx \mathrm{Sp}(1)$. Let $K \approx Z_{2} \oplus Z_{2}$ be a subgroup of $G_{1}$ and consider a point $y \in F(K, M)$ such that $G_{y}$ is locally minimal in this set. Now Borel's formula applied to the action of $K$ implies that $n-\operatorname{dim}_{z_{2}} F(K, M)$ is divisible by 3 . First say that $G_{y}$ is of rank 2. Then we may assume that $y \in F\left(T_{0}, M\right)=F\left(N\left(T_{0}\right), M\right)$ and hence $F(K, M)=F\left(T_{0}, M\right)$ (for otherwise $G_{y}$ would not be locally minimal) is of dimension $n-k-2=n-8$. But $8=n-(n-8)$ is not divisible by 3 so that we must have rank $\left(G_{y}\right) \leqq 1$. If $\operatorname{rank}\left(G_{y}\right)=1$ then it may be assumed that $S_{i} \subset G_{y}$ for some $i=1,2$. The CDT applied to the action of $N\left(S_{i}\right)$ on $F\left(S_{i}, M\right)$ implies that $N\left(S_{i}\right) \cap G_{y}$ is conjugate in $N\left(S_{i}\right)$ to $N\left(S_{i}\right) \cap K_{i}$. Since $K$ is conjugate to a subgroup of $N\left(S_{i}\right)$ this implies that in fact $K$ is conjugate to a subgroup of $K_{i}$ (so that in fact we may assume $G_{y}=K_{i}$ ). Say that $K \subset K_{1}$. Then we can compute

$$
\operatorname{dim}_{z_{2}} F(K, M)=n-k-1+\operatorname{dim} F\left(K, G / K_{1}\right)=n-4
$$

contrary to the above. Thus $K \subset K_{2}$, which is only possible when $S_{2} \subset G_{2}$ (otherwise $K \nsubseteq N\left(K_{2}^{0}\right)$ ). We again compute

$$
\operatorname{dim}_{Z_{2}} F(K, M)=n-k-1+\operatorname{dim} F\left(K, G / K_{2}\right)=n-7 \text { or } n-5,
$$

according as $\operatorname{dim} K_{2}=3$ or 1 , which again is a contradiction. Thus we must have $G_{\nu}$ finite and by Lemma 5.1 we see that $K$ must be conjugate to a subgroup of $H$. This implies that $S_{2} \subset G_{2}$ (since $K$ can be assumed to be in $K_{2}$ and if $S_{2}$ is regular then $\left.K \nsubseteq N\left(K_{2}^{0}\right)\right)$. Thus by a remark above we may assume $H_{1}=H=H_{2}$. However $N\left(S_{2}\right) /\left(N\left(S_{2}\right) \cap K_{2}\right)$ must be a cohomology sphere and, since $K \subset K_{2}$, this can be so only if $K_{2} \cap G_{1} \approx I$. However $H \subset K_{1} \subset N\left(S_{1}\right)$ $=N_{G_{1}}\left(S_{1}\right) \times G_{2}$ and hence $K_{2} / H$ could not possibly be connected, contrary to its being a cohomology sphere. This finishes the proof of our contention that if $S_{1} \subset G_{1}$ then $\operatorname{dim} K_{1}=3$.

Thus in the present case in which $S_{1} \subset G_{1}$ we have $K_{1}^{0}=G_{1}$, and by a remark above we may assume that $H_{1}=H=H_{2} . K_{1} / H$ is a cohomology sphere and it follows that since $K_{1} / H \approx K_{1}^{0} /\left(H \cap K_{1}^{0}\right)$, we have $H \cap G_{1}=(e)$ or $I^{\prime}$. As- 
sume for the moment that $H \cap G_{1}=I^{\prime}$. Then $S_{2}$ must be in $G_{2}$ in order that $H \subset N\left(K_{2}^{0}\right)$. Thus by symmetry we have $K_{2}^{0}=G_{2}$ and $H \cap G_{2}=(e)$ or $I^{\prime}$. If $H \cap G_{2}=(e)$ then we must have $H=I^{\prime} \times(e) \subset G_{1}$ which yields case (7). If $H \cap G_{2}=I^{\prime}$, then $H=I^{\prime} \times I^{\prime}$ which yields case (6). Now assume that $H \cap G_{1}$ $=(e)$. Note that $K_{1}=G_{1} \times\left(K_{1} \cap G_{2}\right)$ and hence

$$
\frac{N\left(S_{1}\right)}{N\left(S_{1}\right) \cap K_{1}} \approx \frac{G_{2}}{K_{1} \cap G_{2}} .
$$

Since this a cohomology sphere we must have $K_{1} \cap G_{2}=(e)$ or $I^{\prime}$. If $K_{1} \cap G_{2}$ $=(e)$ then $H \subset K_{1}=G_{1}$ implies $H=(e)$. If $S_{2} \subset G_{2}$ then by symmetry we see that $K_{2} \supset G_{2}$ and hence $K_{2}=G_{2}$ yielding case (8). If $S_{2}$ is regular then $\operatorname{dim} K_{2}$ $=3$ in order for $N\left(S_{2}\right) /\left(N\left(S_{2}\right) \cap K_{2}\right)$ to be a cohomology sphere (since $K_{2}$ is connected, by $H=(e))$. This yields case (9). If, on the other hand, $K_{1} \cap G_{2}=I^{\prime}$ then we must have $H \approx I^{\prime}$, either in the "diagonal" or in $G_{2}$. If $H \subset G_{2}$ then we must have $K_{2}=G_{2}$ and this yields case (7). If $H$ is in the "diagonal" then since $K_{2} / H$ is a cohomology sphere, we must have $\operatorname{dim} K_{2}=3$ and, in fact, either $K_{2} \approx \mathrm{Sp}(1)$ is diagonal (yielding case (10)) or $K_{2}=I^{\prime} \times \mathrm{Sp}(1)$. We must rule out this latter case. Let $c$ be a central element of $G$ not contained in $H \approx I^{\prime}$. Then we see that $c \in K_{1} \cap K_{2}$ so that $F(c, M) \supset G F\left(S_{1}, M\right) \cup G F\left(S_{2}, M\right)$ $=B$. But also Lemma 5.1 implies that $B \supset F(c, M)$ since $c \notin H$. Hence $F(c, M)$ $=B$. Note that $\operatorname{dim} B=n-7+3=n-4$ and $B$ is separated by $G F\left(T_{0}, M\right)$. But since it may be assumed that $F\left(T_{0}, M\right)=F(G, M)$, we see that $F(c, M)$ is an $(n-4)-\mathrm{cm}_{Z_{2}}$ which is separated by the $(n-8)-\mathrm{cm}_{Z} F\left(T_{0}, M\right)$. This is impossible, and hence this finishes the case $S_{1} \subset G_{1}$.

The only remaining cases are those for which $G=G_{1} \times G_{2}, G_{i} \approx \operatorname{Sp}(1)$ and both $S_{i}$ are regular. First suppose that $G$ is effective, so that $H \cap$ center $(G)$ $=(e)$. Then $N\left(S_{1}\right)=T_{0} \cup s T_{0}$ where $s=s_{1} s_{2}$, and $s_{i}$ is an element of $\left(N_{G_{i}}\left(T_{0} \cap G_{i}\right)-T_{0}\right)$. At most one $K_{i}$ can be 3-dimensional, so say that $\operatorname{dim} K_{1}$ $=1$. Then the fact that $N\left(S_{1}\right) /\left(N\left(S_{1}\right) \cap K_{1}\right)$ is a circle implies that we may assume $K_{1}=N\left(S_{1}\right) \cap K_{1}=\left(T_{0} \cap K_{1}\right) \cup_{s}\left(T_{0} \cap K_{1}\right)$, since $s$ is determined only up to an element of $T_{0}$. Thus $K_{1}$ contains the group $S_{1} \cup_{s} S_{1}$ (which is isomorphic to the nornalizer of a circle subgroup of $\mathrm{Sp}(1)$ ). Thus, since $K_{1} / H_{1}$ is a circle, we must have that $H_{1}$ contains some element $s t, t \in S_{1}$. But then $H_{1}$ contains $(s t)^{2}=s t s t=s^{2} t^{-1} t=s^{2}=s_{1}^{2} s_{2}^{2}$ which is a nontrivial central element of $G$ and consequently $G$ would not be effective.

Now suppose that effectively $G=G_{1} \times G_{2}$ and one of the $G_{i}$, say $G_{1}$, is $\mathrm{SO}(3)$. Let $K \approx Z_{2} \oplus Z_{2}$ be contained in $N_{G_{1}}\left(T_{0} \cap G_{1}\right) \subset G_{1} \approx \mathrm{SO}(3)$. Then, since $K$ cannot normalize any connected rank one group which is not contained in one of the $G_{i}$, we see that $K$ is not conjugate to a subgroup of either $K_{i}$. We may confine our attention to the neighborhood of a point $y$ near which $F\left(T_{0}, M\right)=F(G, M)$. By Lemma 5.1 we see that $F(K, M) \subset B$. Let $z \in F(K, M)$, so that $K \subset G_{z}, \operatorname{dim} G_{z}>0$. By Lemma 5.2 we may assume that a conjugate $g S_{i} g^{-1}$ of one of the $S_{i}$ is contained in $G_{z}$. But then $G_{z}=\left[K G_{z}\right] \supset\left[K g S_{i} g^{-1}\right]$ 
must be of rank two, and since $F\left(T_{0}, M\right)=F(G, M)$ near $y$, we must have that $z \in F(G, M)$. Hence $F(K, M)=F(G, M)$ is an $(n-8)-\mathrm{cm}_{z_{2}}$ near $y$. But by Borel's formula [1, Chapter XIII, 4.3] and the fact that the proper subsubgroups of $K$ are pairwise conjugate in $G$, we see that $8=n-(n-8)$ must be divisible by 3 which is a contradiction.

This completes the proof of our partial classification except for the case in which

$$
G \approx \frac{\operatorname{Sp}(1) \times \operatorname{Sp}(1)}{Z_{2}}
$$

( $Z_{2}$ diagonal)

effectively and both $S_{i}$ are regular, which will take up most of the remainder of this section. We will now restrict our attention, for the time being, to this case, which we shall call case (11) although it will be some time before it is narrowed down to the situation denoted by case (11) in Table 3 . $G$ will be assumed to be effective.

LEMmA 5.3. In case (11), $F(G, M)=F\left(T_{0}, M\right)=F(c, M)$ near $(x)$, where $c$ is the nontrivial central element of $G$.

Proof. Since $c$ must preserve orientation on $M$ and since $G$ is effective, $r=\operatorname{dim}_{z_{2}} F(c, M)=n-2, n-4, n-6$, or $n-8$. To determine $r$ we may restrict our attention to the neighborhood of a point $y$ near which $F(G, M)=F\left(T_{0}, M\right)$. If $r=n-2$ then $G / c \approx \mathrm{SO}(3) \times \mathrm{SO}(3)$ acts on the $n-\mathrm{cm}_{z} M / c$ which was shown above to be impossible since the $S_{i}$ are regular. If $r=n-4$, consider the action of $G / c$ on $F(c, M)$. By Corollary 6.3 we have that the maximum dimension of an orbit is at most three. But the principal isotropy group could not be of maximal rank, since $F\left(T_{0}, M\right)=F(G, M)$ near $y$. Moreover the principal isotropy group must contain a conjugate of one of the $S_{i}$ and hence it must be a "diagonal" $\mathrm{SO}(3)$ in $G / c \approx \mathrm{SO}(3) \times \mathrm{SO}(3)$, since this is the only such group. Moreover $G / c$ has only two types of orbits on $F(c, M)$, fixed points and those with isotropy $\mathrm{SO}(3)$ in the diagonal, since there are no proper subgroups between the diagonal $\mathrm{SO}(3)$ and $G / c \approx \mathrm{SO}(3) \times \mathrm{SO}(3)$. This implies that the subgroup $Z_{2} \oplus Z_{2}$ of one factor of $G / c$ must act freely outside $F(G, M)$ in $F(c, M)$ contrary to Borel's formula. If $r=n-6$ then Corollary 6.3 implies that the maximum dimension of any orbit of $G / c$ on $F(c, M)$ is one. Thus, in fact, $F(c, M)=F(G, M)$ in this case, a contradiction. But we see in the same way that in the only remaining case $r=n-8$ we must have $F(c, M)=F(G, M)$ (even near $x$ ). Since $c \in T_{0} \subset G$ the conclusion follows.

Lemma 5.4. In case (11), $E=\varnothing$ near $x$.

Proof. Let $z \in E$ and let $P$ be a $p$-Sylow subgroup of $G_{z}$ such that $\operatorname{ord}(P) \nmid \operatorname{ord}(H)$. By [1, Chapter VII] there are at most a finite number of nonconjugate $P$ possible, and, since $F(P, M)$ is a $\mathrm{cm}_{z_{p}}$, it is clear that we may assume $\operatorname{dim}_{z_{p}} F(P, M)>\operatorname{dim} F\left(T_{0}, M\right)=n-8$. We may also assume 
that $z$ is in the component of $F(P, M)$ containing $x$ and that if $z^{\prime} \in F(P, M)$ is close to $z$ then $G_{z^{\prime}} \sim G_{z}$. By Lemma 5.1, $N(P)$ is finite, and it follows that, since $F\left(P, G / G_{z}\right)=N(P) G_{z} / G_{z}$, and is of dimension zero, $\operatorname{dim}_{z_{p}} F(P, M)^{*}$ $=\operatorname{dim}_{z_{p}} F(P, M) / N(P)=\operatorname{dim}_{z_{p}} F(P, M) \geqq n-7$ and hence $\operatorname{dim}_{z_{p}} E \geqq n-7$ $+\operatorname{dim} G / G_{z}=n-1$, contrary to Theorem 4 of [5]. Thus $E=\varnothing$ near $x$ as claimed.

Let $G=G_{1} G_{2}, G_{i} \approx \mathrm{Sp}(1)$, be effective, let $q_{i}$ be the nontrivial element in center $G_{i}$ and put $q=q_{1} q_{2}$. Then $G \approx\left(G_{1} \times G_{2}\right) /(e, q)$. Let $T_{i}=G_{i} \cap T_{0}$ and let $s_{i} \in\left(N_{G_{i}}\left(T_{i}\right)-T_{i}\right)$. Put $s=s_{1} s_{2}$. Then $s^{2}=s_{1}^{2} s_{2}^{2}=q_{1} q_{2}=q=e$ in $G$. Clearly $N\left(S_{i}\right)$ $=T_{0} \cup s T_{0}$. At least one of the $K_{i}$, say $K_{1}$, is of dimension one, and therefore $H_{1} \subset K_{1} \subset N\left(S_{1}\right)=T_{0} \cup_{s} T_{0}$. Now if $\operatorname{dim} K_{2}=3$ then we see that, in order for $K_{2} / H_{2}$ to be a cohomology sphere, $H_{2}$ must contain an icosahedral group. Since $H_{1} \sim H_{2}$ does not contain such a group we see that both $K_{i}$ are of dimension one. Since $N\left(S_{i}\right) /\left(N\left(S_{i}\right) \cap K_{i}\right)$ is a circle we must have

$$
K_{i}=N\left(S_{i}\right) \cap K_{i}=J_{i} \cup s t_{i} J_{i}
$$

where $J_{i}=K_{i} \cap T_{0}$ and $t_{i}$ is some element of $T_{0}$ defined up to an element of $J_{i}$. Since $s$ is defined only up to an element of $T_{0}$ we see easily that $s$ can be so chosen that

$$
K_{i}=J_{i} \cup s J_{i}
$$

Moreover, since $K_{i} / H_{i} \approx S^{1}$, we have $H_{i}=H_{i}^{\prime} \cup s j_{i} H_{i}^{\prime}$, where $H_{i}^{\prime}=H_{i} \cap T_{0}$ $\subset J_{i}, J_{i} / H_{i}^{\prime} \approx S^{1}$ and $j_{i} \in J_{i}$. Now all the elements $s t, t \in T_{0}$ are conjugate in $G$. Hence considering the group $K \approx Z_{2} \oplus Z_{2} \subset \mathrm{SO}(3) \subset G$ (diagonally), we have that each $e \neq h \in K$ is conjugate to $s j_{i} \in H_{i}$. Thus for $e \neq h \in K$ we have

$$
\operatorname{dim}_{Z_{2}} F(h, M)=n-6+\operatorname{dim} F\left(s j_{i}, G / H_{i}\right)=n-6+\operatorname{dim} N\left(s j_{i}\right)=n-4 .
$$

Thus by Borel's formula, if $r=\operatorname{dim}_{z_{2}} F(K, M)$, we have

$$
n-r=3((n-4)-r)=3 n-3 r-12
$$

and hence $r=n-6$. Now, since $N(K)$ is finite, it follows easily that $F\left(K, G / G_{z}\right)$ is finite for any $z$. Thus it follows that $\operatorname{dim}_{z_{2}}(F(K, M))^{*}=\operatorname{dim}_{z_{2}} F(K, M)$ $=n-6=\operatorname{dim} M / G$ and hence $F(K, M)$ must touch principal orbits. Therefore we may assume that $K \subset H$.

It is easy to see from the structure of $H$ known so far that, since $H_{1} \sim H_{2}$ in $G, H_{1} \sim H_{2}$ in $N\left(T_{0}\right)$. The verification of this will be left to the reader. Since the only requirements on the $S_{i}$ are that they lie in $T_{0}$ we may, therefore, assume that $H_{1}=H=H_{2}$.

Lemma 5.5. In case (11), if $z \in F\left(S_{i}, M\right)-F\left(T_{0}, M\right)$, then either $G_{z} \sim \mathrm{SO}(3)$ (the "diagonal" of $G$ ) or $G_{z} \sim K_{i}$.

Proof. Let $i=1 . G_{z}$ is of rank one and it is easy to see that if $\operatorname{dim} G_{z}=3$ then, since $S_{1}$ is regular, $G_{z}$ must be "diagonal" and it is also clear that such 
groups must be isomorphic to $\mathrm{SO}(3)$ since they cannot contain center $(G)$ by Lemma 5.3. Assume $\operatorname{dim} G_{z}=1$, so that $G_{z}^{0}=S_{1}$ and $G_{z} \subset N\left(S_{1}\right)$. By the CDT applied to the action of $N\left(S_{1}\right)$ on $F\left(S_{1}, M\right)$ we see that

$$
G_{z}=N\left(S_{1}\right) \cap G_{z} \sim N\left(S_{1}\right) \cap K_{1}=K_{1}
$$

as was to be shown.

Let $C$ be the set of points $z$ for which $\operatorname{dim} G_{z}=3$. Let $z \in C$ and let $U$ be a slice at $z, \operatorname{dim} U=n-3$. If $\operatorname{dim}_{z} F\left(G_{z}, U\right)=n-7$, then by the CDT we would have that $G_{z}$ is locally constant near $z$ on $B$ and this has been shown to be impossible for $\operatorname{dim} G_{z}=3$ in the present case (that is $G_{z}$ could not be a $K_{i}$ ). Thus $\operatorname{dim}_{z} F\left(G_{z}, U\right) \leqq n-8$ and also we must have $\operatorname{dim}_{Z} C \leqq n-5$. Note that if $G_{z} \supset K$ for some $z \in C$ then, since there are only a finite number of subgroups of $G$ isomorphic to $\mathrm{SO}(3)$ and containing $K$, we see that, near $z, F(K, M) \cap C$ is contained in a slice at $z$. Hence $\operatorname{dim}_{z}(F(K, M) \cap C) \leqq n-8$.

It is easily seen that $F\left(H, K_{i} / H\right)$ consists of two points and it follows from the CDT that near $x$ (with $F=F(G, M)=F\left(T_{0}, M\right)$ near $x$ ), $F(H, M-F-C)$ is an $(n-6)-\mathrm{cm}_{z}$. Also $F(K, M)$ is an $(n-6)$-cm over $Z_{2}$ and it follows that $F(K, M)-F-C=F(H, M)-F-C$ since the former is connected. Thus, in particular, $F(K, G / H)=F(H, G / H)$.

Say that $K^{\prime} \sim K$ in $G$ and $K^{\prime} \subset H$. Let $P, P^{\prime}$ be 2-Sylow subgroups of $H$, $P \supset K, P^{\prime} \supset K^{\prime}$. Then there is an element $h \in H$ such that $h P h^{-1}=P^{\prime}$. However, since center $(G) \nsubseteq H$ we see that $K$ is precisely the maximal abelian subgroup of $P$ with elements all of order two. It follows that $h K h^{-1}=K^{\prime}$, that is, $K \sim K^{\prime}$ in $H$. Thus we can compute

$$
F(K, G / H)=\frac{N(K) H}{H} \text { and } F(H, G / H)=\frac{N(H)}{H} .
$$

But $N(K) H \supset N(H)$ so that $N(K) H=N(H)$ and hence $N(K) \subset N(H)$. But there is an element $a \in N(K)$ such that $T_{0} \cap a T_{0} a^{-1}=\operatorname{center}(G)$. Noting that any element of $H$ of order greater than two must be in $T_{0}$, it follows that $H$ contains only elements of order two, and since center $(G) \Phi H$ it follows from the next lemma that

$$
H=K \approx Z_{2} \oplus Z_{2} .
$$

LEMma 5.6. The only subgroup of $G$ containing $K$, not containing center $(G)$ and all of whose elements have order two is $K$ itself.

Proof. Let $K^{\prime}$ satisfy the hypotheses. Since center $G \nsubseteq K^{\prime}$ the map $f: G \rightarrow G /$ center $G \approx L_{1} \times L_{2}=L$ (which defines the $L^{\prime}$ 's), $L_{i} \approx \mathrm{SO}(3)$ is an isomorphism on $K^{\prime}$. We also know that $K^{\prime} \cap G_{i}=(e)$ and it follows easily that that $f\left(K^{\prime}\right) \cap L_{i}=(e)$ also. Thus the map $f^{\prime}: L \rightarrow L_{1}$ is also an isomorphism on $f\left(K^{\prime}\right)$ and it follows from known facts about $\mathrm{SO}(3)$ that $K^{\prime}$ is either $\approx Z_{2}$ or $\approx Z_{2} \oplus Z_{2}$. Since $K^{\prime} \supset K \approx Z_{2} \oplus Z_{2}$ we must have $K^{\prime}=K$ as claimed. 
REMARK. It can be shown that if the hypothesis $K^{\prime} \supset K$ is removed from Lemma 5.6 then the group $K^{\prime}$ is conjugate to a subgroup of $K$.

Note that we now have that case (11) is as shown in Table 3, since the fact that $K_{i}=S_{i} H$ follows from the fact that $K_{i} / H \approx S^{1}$. The fact that $K_{i} \approx \mathrm{O}(2)$ follows from the fact that $S_{i} \cap H=T_{0} \cap H$. (If $S_{i} \cap H \neq T_{0} \cap H$ then $K_{i} \cap T_{0}$ contains $Z_{2} \oplus Z_{2} \subset T_{0}$ which contains $c$ contrary to Lemma 5.3.)

Lemma 5.7. In case (11), the natural map $F(H, M) / N(H) \rightarrow M / G$ is a homeomorphism near $x$.

Proof. This is equivalent to $F\left(H, G / G_{z}\right)=N(H) G_{z} / G_{z}$ for any $G_{z} \supset H$, and this would follow if we knew that whenever $H^{\prime} \sim H$ in $G, H^{\prime} \subset G_{z}$ then $H^{\prime} \sim H$ in $G_{z}$. To see this we may assume that $\operatorname{rank}\left(G_{z}\right)=1$, for otherwise $G_{z}=H$ or $G$. In this case we know that either $G_{z} \sim K_{i} \approx \mathrm{O}(2)$, or $G_{z} \approx \mathrm{SO}(3)$ by Lemma 5.5. But in these cases the result is clear since all groups isomorphic to $Z_{2} \oplus Z_{2}$ are conjugate in these subgroups of $G$.

We shall now show that there is a cross-section in $F(H, M)$ for the fibre bundle

$$
M-B \stackrel{G / H}{\longrightarrow} \frac{M-B}{G} .
$$

Note that $N(H) / H \approx Z_{2} \oplus \sigma_{3}$. Let $J \subset N(H) / H, J \approx Z_{3}$, and $K=(N(H) / H) / J$ $\approx Z_{2} \oplus Z_{2}$ (not to be confused with our previous use of the letter $K$ ). Consider the orbit maps

$$
F(H, M) \stackrel{J}{\longrightarrow} \frac{F(H, M)}{J} \stackrel{K}{\longrightarrow} \frac{F(H, M)}{N(H)} \approx M / G .
$$

We know, by [1, Chapter III, 2.3], that $F(H, M) / J$ is an $(n-6)-\mathrm{cm}_{z_{2}}$. Also, since $K$ acts effectively on $F(H, G / H) / J$ and hence on $F(H, M) / J$, we must have, by Borel's formula, that $\operatorname{dim}_{z_{2}}(F(K, F(H, M) / J))=n-8$ and that there is an element $g \in K$ with a fixed point set of dimension $n-7$. Lemma 2.5 applied to the action of $K / g$ on $(F(H, M) / J) / g$ yields that $M / G$ is an $(n-6)-\mathrm{cm}_{z_{2}}$ with boundary consisting of the exceptional orbits of the above action of $K$.

Note that if $G_{z} \supset H$ is an isotropy group of a singular orbit of $G$ on $M$, then $\left(\left(G_{z} \cap N(H)\right) / H\right) / J$ is not trivial so that we see that the boundary of $M / G \approx F(H, M) / N(H)$ is precisely $B^{*}$. In particular, $B^{*}=B / G$ is an $(n-7)$-cm over $Z_{2}$.

Lemma 2.5 also implies that $F(H, M-B) / J$ is the union of four disjoint copies of $F(H, M-B) / N(H) \approx(M-B) / G$. Since $(M-B) / G$ is an $(n-6)-\mathrm{cm}$ over $Z$ we have only to show (by 6.5 ) that it is orientable over $Z_{3}$ and that $H_{c}^{n-7}\left((M-B) / G, Z_{3}\right)={ }_{L x} 0$, in order to conclude that the map $F(H, M-B)$ $\rightarrow(M-B) / G$ has a cross-section near $x$. 
Let $F=F(G, M)$ and $B=F \cup C \cup D_{1} \cup D_{2}$, where $D_{i}$ consists of points with isotropy groups conjugate to $K_{i}$. We know $\operatorname{dim} F \cup C \leqq n-5$ and $G / K_{i}$ is easily seen to be nonorientable (since conjugation by an element of $K_{i}-S_{i}$ reverses the orientation of $\left.G / S_{i}\right)$. Thus for the spectral sequence with coefficients in $Z_{3}$ of the fibering $D_{i} \rightarrow D_{i}^{*}$ we have

$$
E_{2}^{n-7,5} \approx H_{c}^{n-7}\left(D_{i}^{*}, H^{5}\left(G / K_{i}, Z_{3}\right)\right)=0
$$

since the coefficients are trivial. Thus $H_{c}^{n-2}\left(D_{i}, Z_{3}\right)=E_{\infty}^{n-7,5}=0$, and consequently $H_{c}^{n-2}\left(B, Z_{3}\right)=0$. This implies that $H_{c}^{n-1}\left(M-B, Z_{3}\right)={ }_{L x} 0$. But for the spectral sequence (coefficients in $Z_{3}$ ) of $M-B \rightarrow(M-B) / G$ we have (by [1, Chapter XV, Lemma 6.2])

$$
0={ }_{L x} H_{c}^{n-1}\left(M-B, Z_{3}\right) \approx E_{\infty}^{n-7,6} \approx H_{2}^{n-7,6} \approx H_{c}^{n-7}\left(\frac{M-B}{G}, Z_{3}\right)
$$

since $H^{5}\left(G / H, Z_{3}\right) \approx H_{1}\left(G / H, Z_{3}\right)=0$. Moreover,

$$
H_{c}^{n-6}\left(\frac{M-B}{G}, Z_{3}\right) \approx E_{2}^{n-6,6} \approx E_{\infty}^{n-6,6} \approx H_{c}^{n}\left(M-B, Z_{3}\right)=Z_{3}
$$

so that $(M-B) / G$ is orientable as was to be shown.

We will now investigate the structure of $C$ in order to show eventually that $C=\varnothing$. Let $z \in C$ and assume, as we may, that $K_{1} \subset G_{z}$. We will first show that $\operatorname{dim}_{L}(C, z)=n-5$ for any $L$. Let $U$ be a slice at $z, \operatorname{dim} U=n-3$. Then, since $\operatorname{dim}\left(S_{1}, G_{z} / K_{1}\right)=0$ we have that $\operatorname{dim}_{z} F\left(S_{1}, U\right)=n-7$. Note that it will suffice to show $\operatorname{dim}_{z_{2}} F\left(G_{z}, U\right)=n-8$.

There are exactly three types of orbits of $G_{z}$ on $U$, namely fixed points, principal orbits (isotropy group $H \approx Z_{2} \oplus Z_{2}$ ), and projective planes (isotropy group $N_{G_{z}}\left(S_{1}\right)$ ). We will use the notation $G^{\prime}=G_{z}, B^{\prime}=B \cap U, F^{\prime}=F\left(G^{\prime}, U\right)$ $=C \cap U$. Note that $F\left(S_{1}, U\right)=F\left(N_{G^{\prime}}\left(S_{1}\right), U\right)$ so that $B^{\prime} / G^{\prime} \approx F\left(S_{1}, U\right)$ is an $(n-7)$-cm over $Z$ and also the set $B^{\prime}-F^{\prime}$ of points on projective plane orbits has a cross-section $F\left(S_{1}, B^{\prime}-F^{\prime}\right)$. As seen above there is also a cross-section in $F(H, M-B)$ for the principal orbits of $G$ and it follows that there is a cross-section in $F\left(H, U-B^{\prime}\right)$ for the principal orbits of $G^{\prime}$ on $U$ so that

$$
U-B^{\prime} \approx \frac{U-B^{\prime}}{G} \times G^{\prime} / H ; \quad B^{\prime}-F^{\prime} \approx \frac{B^{\prime}-F^{\prime}}{G^{\prime}} \times G^{\prime} / N_{G^{\prime}}\left(S_{1}\right) .
$$

Now say that $\operatorname{dim}_{z_{2}}\left(F^{\prime}\right)<n-8$ near $z$. Then $F^{\prime}$ cannot separate the $(n-7)-\mathrm{cm}_{Z_{2}} B^{\prime} / G^{\prime}$ locally. Thus $H_{c}^{n-7}\left(\left(B^{\prime}-F^{\prime}\right) / G^{\prime}, Z_{2}\right)={ }_{L_{2}} Z_{2}$. Considering the Leray spectral sequence of the map $B^{\prime} \rightarrow B^{\prime} / G^{\prime}$ we see that $E_{2}^{n-7,2}$ $\approx H_{c}^{n-7}\left(B^{\prime} / G^{\prime}, \quad \mathcal{H}^{2}\left(G^{\prime} / G_{y}^{\prime}, \quad Z_{2}\right)\right)=H_{c}^{n-7}\left(\left(B^{\prime}-F^{\prime}\right) / G^{\prime}, \quad H^{2}\left(P^{2}, \quad Z_{2}\right)\right)$ $=H_{c}^{n-7}\left(\left(B^{\prime}-F^{\prime}\right) / G^{\prime}, Z_{2}\right)={ }_{L_{2}} Z_{2}$. Thus $H_{c}^{n-5}\left(B^{\prime}, Z_{2}\right)={ }_{L_{2}} Z_{2}$ which implies that $Z_{2}={ }_{L z} H_{c}^{n-4}\left(U-B^{\prime}, Z_{2}\right)={ }_{L_{z}} H_{c}^{n-6}\left(\left(U-B^{\prime}\right) / G^{\prime}, Z_{2}\right) \otimes H_{c}^{2}\left(G^{\prime} / H, Z_{2}\right) \approx Z_{2} \oplus Z_{2}$ 
and this contradiction implies that we must have had $\operatorname{dim}_{Z_{2}}\left(F^{\prime}, z\right)=n-8$ as claimed.

It now follows from Case II applied to the action of $G^{\prime}$ on $U$ that $F^{\prime}=F\left(G_{z}, U\right)$ is an $(n-8)-\mathrm{cm}_{z_{2}}$. Since $C$ is locally a product of $F\left(G_{z}, U\right)$ by a 3-cell, we have that $C$ is an $(n-5)$-cm over $Z_{2}$. Also $C / G$ is an $(n-8)$-cm over $Z_{2}$.

Consider $H_{c}^{n-7}\left((B-F-C) / G, Z_{2}\right)$, whose rank is the number of components of the $(n-7)-\mathrm{cm}(B-F-C) / G$. We have $H_{c}^{n-7}\left((B-F-C) / G, Z_{2}\right)$ $\approx H_{c}^{n-2}\left(B-X-C, Z_{2}\right) \approx H_{c}^{n-2}\left(B, Z_{2}\right)={ }_{L x} H_{c}^{n-1}\left(M-B, Z_{2}\right)={ }_{L x} H^{5}\left(G / H, Z_{2}\right)$ $\approx H_{1}\left(G / H, Z_{2}\right) \approx Z_{2} \oplus Z_{2}$, since $M-B \approx(M-B) / G \times G / H$ and $M / G$ is an $(n-6)-\mathrm{cm}_{Z_{2}}$ with boundary $B / G$.

Moreover we know that $H_{c}^{n-8}\left((B-F) / G, Z_{2}\right)={ }_{L x} 0$ and $H_{c}^{n-7}\left((B-F) / G, Z_{2}\right)$ $={ }_{L x} Z_{2} \oplus Z_{2}$, since

$$
\frac{B-F}{G} \approx \bigcup_{i} \frac{F\left(S_{i}, M\right)-F\left(T_{0}, M\right)}{N\left(S_{i}\right)} .
$$

Thus there are neighborhoods $V_{1} \subset V_{2} \subset V_{3}$ of $x$ such that, with $B_{i}=B \cap V_{i}$, $C_{i}=C \cap V_{i}$, we have that in the diagram (coefficients in $Z_{2}$ )

$$
\begin{gathered}
H_{c}^{n-8}\left(\frac{B_{1}-F}{G}\right) \stackrel{j_{i}}{\rightarrow} H_{c}^{n-8}\left(\frac{C_{1}}{G}\right) \stackrel{d_{1}}{\rightarrow} H_{c}^{n-7}\left(\frac{B_{1}-F-C}{G}\right) \stackrel{i_{1}}{\rightarrow} H_{c}^{n-7}\left(\frac{B_{1}-F}{G}\right) \rightarrow 0 \\
\downarrow \begin{array}{l}
\downarrow h_{1}^{\prime} \\
\qquad f_{1}
\end{array} \\
H_{c}^{n-8}\left(\frac{B_{2}-F}{G}\right) \stackrel{j_{2}}{\rightarrow} H_{c}^{n-8}\left(\frac{C_{2}}{G}\right) \stackrel{d_{2}}{\rightarrow} H_{c}^{n-7}\left(\frac{B_{2}-F-C}{G}\right) \stackrel{i_{2}}{\rightarrow} H_{c}^{n-7}\left(\frac{B_{2}-F}{G}\right) \rightarrow 0 \\
\downarrow \downarrow h_{2}^{\prime} \\
H_{c}^{n-8}\left(\frac{B_{3}-F}{G}\right) \stackrel{j_{3}}{\rightarrow} H_{c}^{n-8}\left(\frac{C_{3}}{G}\right) \stackrel{d_{3}}{\rightarrow} H_{c}^{n-7}\left(\frac{B_{3}-F-C}{G}\right) \stackrel{i_{3}}{\rightarrow} H_{c}^{n-7}\left(\frac{B_{2}-F}{G}\right) \rightarrow 0
\end{gathered}
$$

$h_{1}^{\prime}=0=h_{2}^{\prime}, \operatorname{Im} g_{1} \approx Z_{2} \oplus Z_{2}$ and is mapped isomorphically by $g_{2}$ onto $\operatorname{Im} g_{2}$, and similarly with $h_{1}$ and $h_{2}$.

Thus $Z_{2} \oplus Z_{2} \approx \operatorname{Im} h_{1}=\operatorname{Im} h_{1} i_{1}=\operatorname{Im} i_{2} g_{1}$ and, since $\operatorname{Im} g_{1} \approx Z_{2} \oplus Z_{2}, i_{2}$ must map $\operatorname{Im} g_{1}$ isomorphically. Hence if $\alpha \in H_{c}^{n-8}\left(C_{1} / G\right)$ we have that $i_{2} g_{1} d_{1}(\alpha)$ $=i_{2} d_{2} f_{1}(\alpha)=0$ implies that $0=g_{1} d_{1}(\alpha)=d_{2} f_{1}(\alpha)$. Thus $f_{1}(\alpha)=j_{2}(\beta)$ for some $\beta$ and $f_{2} f_{1}(\alpha)=f_{2} j_{2}(\beta)=j_{3} h_{2}^{\prime}(\beta)=0$ since $h_{2}^{\prime}=0$. Thus $H_{c}^{n-8}\left(C / G, Z_{2}\right)={ }_{L x} 0$, and, since $C / G$ is an $(n-8)$-cm over $Z_{2}$, it follows that $C=\varnothing$ near $x$ as was to be shown.

We shall now finish the proof of Case III. Using Lemma 5.1 we see that $E=\varnothing$ near $x$ in cases (1), (2), (3), (4), (5), (8), and (9). This has also been seen in case (11). This also follows in case (6) since there are no groups contained properly between $I$ and $\mathrm{SO}(3)$, and using this fact together with Lemma 5.1 we see that this is true in case (7). In case (10) we see that any 
subgroup properly containing $H$ must intersect a factor $G_{i}$ of $G$ and using Lemma 5.1 we see that $E=\varnothing$ near $x$ in this case also, so that this is true in general.

In case (11) we have seen that the principal orbits have a cross-section in $F(H, M)$. In the other cases, except for (3) and (10) we see that $N(H) / H$ is connected and hence acts trivially on $H^{*}(G / H)$. In case (10), $N(H) / H \approx Z_{2}$ and is represented by the central element of $G$, so that, as may easily be seen, its action on $H^{*}(G / H)$ is trivial. Considering case (3), we note that $G / H$ $\approx \mathrm{SO}(3) \times_{Z_{2}} S^{1}$ (in the notation of [1, Chapter IV, 1.3]) is an SO(3)-bundle over $S^{1}$ and hence the groups $H^{i}(G / H, Z)$ are $Z, Z, Z_{2},\left(Z \oplus Z_{2}\right)$, and $Z$, for $i=0,1,2,3$, and 4 respectively. Hence $N(H)$ acts trivially on this if and only if it does so on $H^{i}(G / H, Q)\left(Q=\right.$ rationals). However $\pi^{*}: H^{*}(G / H) \rightarrow H^{*}(G)$ is a rational isomorphism and the action of $N(H)$ on $H^{*}(G)$ is trivial, since it is just right translation. Thus, in general, the structural group of $M-B$ $\rightarrow(M-B) / G$ acts trivially on $H^{*}(G / H, Z)$.

We have that $F\left(T_{0}, M\right)=F(G, M)$ in all cases except possibly (3), since if $z \in F\left(T_{0}, M\right)$ then $G_{z} \supset K_{1} \cup K_{2}$ (up to conjugation of the $K_{i}$ ) and the only such group is $G$ in these cases (except (11) for which this has already been shown). In case (3), $F\left(G_{2}, M\right)=F\left(T_{0}, M\right)$ by Lemma 5.2 , so that $G_{1}$ acts on the $\operatorname{cm}_{Z} F\left(G_{2}, M\right)$ with an open set of fixed points. Thus $F(G, M)$ $=F\left(G_{1}, F\left(G_{2}, M\right)\right)=F\left(G_{2}, M\right)=F\left(T_{0}, M\right)$ in this case also.

Moreover, Corollary 2.4 implies easily that if $G_{z} \supset K_{i}, \operatorname{dim} G_{z}=\operatorname{dim} K_{i}$, then $G_{z}=K_{i}$. But then if $G_{z} \nsim G$ or $H$ then rank $\left(G_{z}\right)=1$ and, using special facts already shown in cases (3) and (11), it follows that $G_{z}$ must be conjugate to one of the $K_{i}$. Thus there are exactly four orbit types near $x$.

Since $B / G$ is the union

$$
\frac{F\left(S_{1}, M\right)}{N\left(S_{1}\right)} \cup \frac{F\left(S_{2}, M\right)}{N\left(S_{2}\right)}
$$

of two $(n-k-1)$-cms with common boundary $F(G, M)=F\left(T_{0}, M\right)$, an $(n-k-2)$-cm over $Z$, it follows that $B / G$ is an $(n-k-1)-\mathrm{cm}_{z}$. Considering the maps

$$
F\left(K_{i}, M\right)-F(G, M) \rightarrow \frac{F\left(K_{i}, M\right)-F(G, M)}{N\left(K_{i}\right) / K_{i}} \approx \frac{F\left(S_{i}, M\right)-F(G, M)}{N\left(S_{i}\right)}
$$

we obtain, by using Theorems 6.4 and 6.5 , that these maps have cross-sections. That is, we note that in all cases $\left(N\left(K_{i}\right) / K_{i}\right) /\left(N\left(K_{i}\right) / K_{i}\right)^{0}$ is solvable and we apply 6.5 to the map $F\left(K_{i}, M-F\right) /\left(N\left(K_{i}\right) / K_{i}\right)^{0}$ $\rightarrow F\left(K_{i}, M-F\right) / N\left(K_{i}\right) / K_{i}$ and then apply 6.4 to the map $F\left(K_{i}, M-F\right)$ $\rightarrow F\left(K_{i}, M-F\right) /\left(N\left(K_{i}\right) / K_{i}\right)^{0}$ (or rather to the part over a component of the latter space).

Letting $B-F=D_{1} \cup D_{2}$ where $D_{1}=\left\{z \mid G_{z} \sim K_{i}\right\}$ we now have that $D_{i} \approx D_{i} / G \times G / K_{i}$ and $D_{i} / G \approx F\left(S_{i}, M\right) / N\left(S_{i}\right)-F(G, M)$. Now, considering 
the Leray spectral sequence of the map $B \rightarrow B / G$, we see that $E_{2}^{p, 0}$ $\approx H_{c}^{p}(B / G, Z)={ }_{L x} 0$ for $p \leqq n-k-2$. Also for $q>0, E_{2}^{p, q} \approx H_{c}^{p}\left(D_{1} / G, H^{q}\left(G / K_{1}\right)\right)$ $\oplus H_{c}^{p}\left(D_{2} / G, H^{q}\left(G / K_{2}\right)\right)={ }_{L x} 0$ for $p \leqq n-k-2$, since the coefficients are constant.

It follows that $H_{c}^{t}(B, Z)={ }_{L x} 0$ for $i \leqq n-k-2$ and hence also $H_{c}^{i}(M-B, Z)$ $={ }_{L x} 0$ for $i \leqq n-k-1$. Since, as seen above, the structural group of the fibering $M-B \rightarrow(M-B) / G$ acts trivially on $H^{*}(G / H, Z)$ we have by Theorem 6.6 that $H_{c}^{t}((M-B) / G, Z)={ }_{L x} 0$ for $i \leqq n-k-1$. Also $H_{c}^{n-k}((M-B) / G, Z)$ $=E_{2}^{n-k, k}=E_{\infty}^{n-k, k}=H_{c}^{n}(M-B, Z) \approx Z$. Moreover $M / G-F$ is an $(n-k)-\mathrm{cm}_{Z}$ with boundary $B / G-F$ by the CDT and thus, since $B / G$ is an $(n-k-1)-\mathrm{cm}_{Z}$, we have by Lemma 2.6 that $M / G$ is an $(n-k)-\mathrm{cm}_{z}$ with boundary $B / G$, which completes the proof of Case III.

6. Appendix. We shall give here the statements of some theorems for which proofs appear (essentially) in the literature but the existing statements of which are not sufficiently general for our purposes. The notation is as in the present paper.

Theorem 6.1 (Montgomery [1, Chapter IX, 2.1]; YANG [7, Theorem 2]). Let $G$ be a compact Lie group acting on an $n$-cm $M$ over $K_{p}$ (where $K_{p}$ denotes a field of characteristic $p$, possibly zero). Then $\operatorname{dim}_{K_{p}} B \leqq n-2$.

Theorem 6.2 (Montgomery [1, Chapter IX, 2.2]; YANG [7, Theorem 2]). Let $G$ be a compact Lie group acting on an $n-c m M$ over $K_{p}$, and let $t$ be an integer $0 \leqq t<k$. Then the union of all orbits of dimension $\leqq t$ is a closed set of dimension $\leqq n-k+t-1$ over $K_{p}$.

Corollary 6.3 (Montgomery [1, Chapter IX, 2.2 Corollary]). Let $G$ and $M$ be as above, then $\operatorname{dim}_{K_{p}} M / G=n-k$ (near any point) and $\operatorname{dim}_{K_{p}} B / G$ $\leqq n-k-1$.

These three results are essentially the same as those cited except that $K_{p}$ replaces $Z$ as coefficients. The proofs are almost the same, with $K_{p}$ in place of $Z$, and, in the proof of [1, Chapter IX, 2.1], the group $H$ should be taken to be a circle group such that $H \cap G_{y}$ is finite, the reference to Chapter V, 2.6 being replaced by a reference to $[1$, Chapter $\mathrm{V}, 3.2]$. The equality $\operatorname{dim}_{K_{p}} M / G$ $=n-k$ follows from the fact that locally near some orbit of dimension $k, M$ is the product of the orbit with $M / G$. (Hence $\operatorname{dim}_{K_{p}} M / G \geqq n-k$ and the reverse inequality follows from the proof of [1, Chapter IX, 2.2].)

THEOREM 6.4. Let $G$ be a compact connected Lie group acting on a locally separable, locally compact space $M$ of finite covering dimension. Assume that $G$ acts freely outside the closed set $A$, that $(M-A) / G$ is an $m-c m$ over $Z$, and that

$$
H_{c}^{i}\left(\frac{M-A}{G}, Z\right)={ }_{L x^{*}}\left\{\begin{array}{ll}
Z, & i=m, \\
0, & i \neq m,
\end{array} \quad \text { for some } x \in A .\right.
$$

Then there is a local cross-section for the orbits of $G$ on $M-A$ near $x$. 
This follows from the proof of [1, Chapter XV, 3.3].

TheOREM 6.5. Let $G$ be a finite solvable group acting on a space $X$ freely outside the closed set $A \subset X$. Say that $x^{*} \in A^{*}$ has a fundamental system of neighborhoods $Y_{\alpha}$ such that each $Y_{\alpha}-A / G$ is connected. Assume further that $(X-A) / G$ is an orientable $n-c m$ over $Z_{p}$ for all $p \mid \operatorname{ord}(G)$ and that $H_{c}^{n-1}\left((X-A) / G, Z_{p}\right)={ }_{L x} 0$ for all $p \mid \operatorname{ord}(G)$. Then there is a local cross-section near $x$ for the orbits of $G$ on $X-A$.

This follows from the proof of [1, Chapter XV, 3.4].

THEOREM 6.6. Let $G$ be a compact connected Lie group acting on a space $X$ and let $A$ be a closed invariant subset of $X$ such that the orbits of $G$ in $X-A$ are all of the same type $G / H$. Let $L$ be a principal ideal ring and suppose that the structural group of the fibering

$$
X-A \stackrel{G / H}{\longrightarrow} \frac{X-A}{G}
$$

acts trivially on $H^{*}(G / H, L)$. Let $x \in F(G, X) \subset A$ and say that $H_{c}^{i}(X-A, L)$ $={ }_{L x} 0$ for all $i \leqq i_{0}$. Then also $H_{c}^{i}((X-A) / G, L)={ }_{L x}{ }^{*} 0$ for all $i \leqq i_{0}$.

This essentially follows from the proof of $[1$, Chapter XV, 6.3].

\section{REFERENCES}

1. A. Borel, Seminar on transformation groups, Annals of Mathematics Studies, no. 46, 1960.

2. G. E. Bredon, Orientation in generalized manifolds and applications to the theory of transformation groups, Michigan Math. J. vol. 7 (1960) pp. 35-64. 118.

3. - - Some theorems on transformation groups, Ann. of Math. vol. 67 (1958) pp. 104-

4. H. Cartan and S. Eilenberg, Homological algebra, Princeton University Press, 1956.

5. D. Montgomery and C. T. Yang, Orbits of highest dimension, Trans. Amer. Math. Soc. vol. 87 (1958) pp. 284-293.

6. R. G. Swan, Groups with periodic cohomology, Bull. Amer. Math. Soc. vol. 65 (1959) pp. 368-370.

7. C. T. Yang, Transformation groups on a homological manifold, Trans. Amer. Math. Soc. vol. 87 (1958) pp. 261-283.

8. H. J. Zassenhaus, The theory of groups, New York, Chelsea Publishing Co., 1958.

InSTITUTe for Advanced Study,

Princeton, New Jersey 